TRANSACTIONS OF THE

AMERICAN MATHEMATICAL SOCIETY

Volume 355, Number 9, Pages 3727-3759

S 0002-9947(03)03315-4

Article electronically published on May 29, 2003

\title{
THE PERIODIC EULER-BERNOULLI EQUATION
}

\author{
VASSILIS G. PAPANICOLAOU
}

Abstract. We continue the study of the Floquet (spectral) theory of the beam equation, namely the fourth-order eigenvalue problem

$$
\left[a(x) u^{\prime \prime}(x)\right]^{\prime \prime}=\lambda \rho(x) u(x), \quad-\infty<x<\infty,
$$

where the functions $a$ and $\rho$ are periodic and strictly positive. This equation models the transverse vibrations of a thin straight (periodic) beam whose physical characteristics are described by $a$ and $\rho$. Here we develop a theory analogous to the theory of the Hill operator $-(d / d x)^{2}+q(x)$.

We first review some facts and notions from our previous works, including the concept of the pseudospectrum, or $\psi$-spectrum.

Our new analysis begins with a detailed study of the zeros of the function $F(\lambda ; k)$, for any given "quasimomentum" $k \in \mathbb{C}$, where $F(\lambda ; k)=0$ is the Floquet-Bloch variety of the beam equation (the Hill quantity corresponding to $F(\lambda ; k)$ is $\Delta(\lambda)-2 \cos (k b)$, where $\Delta(\lambda)$ is the discriminant and $b$ the period of $q$ ). We show that the multiplicity $m\left(\lambda^{*}\right)$ of any zero $\lambda^{*}$ of $F(\lambda ; k)$ can be one or two and $m\left(\lambda^{*}\right)=2$ (for some $k$ ) if and only if $\lambda^{*}$ is also a zero of another entire function $D(\lambda)$, independent of $k$. Furthermore, we show that $D(\lambda)$ has exactly one zero in each gap of the spectrum and two zeros (counting multiplicities) in each $\psi$-gap. If $\lambda^{*}$ is a double zero of $F(\lambda ; k)$, it may happen that there is only one Floquet solution with quasimomentum $k$; thus, there are exceptional cases where the algebraic and geometric multiplicities do not agree.

Next we show that if $(\alpha, \beta)$ is an open $\psi$-gap of the pseudospectrum (i.e., $\alpha<\beta$ ), then the Floquet matrix $T(\lambda)$ has a specific Jordan anomaly at $\lambda=\alpha$ and $\lambda=\beta$.

We then introduce a multipoint (Dirichlet-type) eigenvalue problem which is the analogue of the Dirichlet problem for the Hill equation. We denote by $\left\{\mu_{n}\right\}_{n \in \mathbb{Z}}$ the eigenvalues of this multipoint problem and show that $\left\{\mu_{n}\right\}_{n \in \mathbb{Z}}$ is also characterized as the set of values of $\lambda$ for which there is a proper Floquet solution $f(x ; \lambda)$ such that $f(0 ; \lambda)=0$.

We also show (Theorem 7) that each gap of the $L^{2}(\mathbb{R})$-spectrum contains exactly one $\mu_{n}$ and each $\psi$-gap of the pseudospectrum contains exactly two $\mu_{n}$ 's, counting multiplicities. Here when we say "gap" or " $\psi$-gap" we also include the endpoints (so that when two consecutive bands or $\psi$-bands touch, the in-between collapsed gap, or $\psi$-gap, is a point). We believe that $\left\{\mu_{n}\right\}_{n \in \mathbb{Z}}$ can be used to formulate the associated inverse spectral problem.

As an application of Theorem 7 , we show that if $\nu^{*}$ is a collapsed ("closed") $\psi$-gap, then the Floquet matrix $T\left(\nu^{*}\right)$ is diagonalizable.

Some of the above results were conjectured in our previous works. However, our conjecture that if all the $\psi$-gaps are closed, then the beam operator is the square of a second-order (Hill-type) operator, is still open.

Received by the editors November 13, 2001 and, in revised form, November 10, 2002. 2000 Mathematics Subject Classification. Primary 34B05, 34B10, 34B30, 34L40, 74B05.

Key words and phrases. Euler-Bernoulli equation for the vibrating beam, beam operator, Hill operator, Floquet spectrum, pseudospectrum, algebraic/geometric multiplicity, multipoint eigenvalue problem. 


\section{INTRODUCTION}

The term "periodic Euler-Bernoulli equation" refers to the eigenvalue problem

$$
\left[a(x) u^{\prime \prime}(x)\right]^{\prime \prime}=\lambda \rho(x) u(x), \quad-\infty<x<\infty,
$$

where $a(x)$ and $\rho(x)$ are strictly positive and periodic with a common period $b$, satisfying the smoothness conditions $a \in C^{2}(\mathbb{R})$ and $\rho \in C(\mathbb{R})$. Furthermore, without loss of generality, $a(x)$ and $\rho(x)$ are normalized so that

$$
\int_{0}^{b}\left[\frac{\rho(x)}{a(x)}\right]^{1 / 4} d x=b
$$

One advantage of this normalization is that the asymptotics of certain quantities, such as $|\lambda| \rightarrow \infty$, become simpler, and this is the only reason that (2) is used in the present work (see Section 3).

The study of (1) was initiated by the author in 31] and 33. There are theoretical as well as practical reasons for studying (11). The Floquet (spectral) theory of (1) is richer than its second-order counterpart (namely the Sturm-Liouville problem with periodic coefficients, also known as Hill's equation, or the one-dimensional Schrödinger equation with a periodic potential). All the main second-order properties continue to hold, while new interesting phenomena arise which are nonexistent in the second-order case. On the practical side, we notice that a typical application of (1I) is that it models the transverse vibrations of a thin straight beam with periodic characteristics (see, e.g., [36] or [17]). Elastic structures consisting of many thin elements arranged periodically are quite common. Although there are some authors who have studied such problems numerically (see, for example, [28]), as far as we know, [31] and [33] are the only theoretical works on (11). However, recently there is an increasing interest in higher-order periodic eigenvalue problems (e.g., [2], [6], 7]). For results on the second-order inverse periodic problem, or higher-order nonperiodic inverse problems, the reader may see, e.g., [3], 4], [5], 8], 10], [14, [15], [17, [23, 24], 25], 26], 27, [32, [35, [38.

The present work continues the investigation on the Floquet spectral theory of (1), initiated in [31] and 33. The goal is a theory analogous to the theory of the Hill operator $-(d / d x)^{2}+q(x)$.

In Section 2 we review some facts and notions from our previous works, including the concept of the pseudospectrum, or $\psi$-spectrum, introduced in [33. Theorems numbered here by capital Latin letters have been proved in our previous works [31] and 33. At the end of Section 2 we have included a subsection containing some ideas on the significance of the pseudospectrum.

The new analysis begins in Section 3. We first describe (in Subsection 3.1) a technique we use for proving certain theorems (Theorems 2 and 7 of this work). Then, in Subsection 3.2 we give a detailed study of the zeros of the function $F(\lambda ; k)$, in the spirit of [16, for any given "quasimomentum" $k \in \mathbb{C}$, where $F(\lambda ; k)=0$ is the Floquet-Bloch variety of the beam equation (the Hill quantity corresponding to $F(\lambda ; k)$ is $\Delta(\lambda)-2 \cos (k b)$, where $\Delta(\lambda)$ is the discriminant of the Hill operator and $b$ the period of $q$ ). We show that the multiplicity $m\left(\lambda^{*}\right)$ of any zero $\lambda^{*}$ of $F(\lambda ; k)$ can be one or two and $m\left(\lambda^{*}\right)=2$ (for some $k$ ) if and only if $\lambda^{*}$ is also a zero of another entire function $D(\lambda)$, independent of $k$. Furthermore, we show that $D(\lambda)$ has exactly one zero in each gap of the spectrum and two zeros (counting multiplicities) in each $\psi$-gap. If $\lambda^{*}$ is a double zero of $F(\lambda ; k)$ it may happen 
that there is only one Floquet solution with quasimomentum $k$; thus, there are exceptional cases where the algebraic and geometric multiplicities do not agree.

In Section 4 we first (Subsection 4.1) review briefly some facts regarding certain operators $L_{k}$, where $k \in \mathbb{C}$ is the quasimomentum, and then, in Subsection 4.2, we apply them to show that if $(\alpha, \beta)$ is an open $\psi$-gap of the pseudospectrum (i.e., $\alpha<\beta$ ), then the Floquet matrix $T(\lambda)$ has a specific Jordan anomaly at $\lambda=\alpha$ and $\lambda=\beta$ (this was conjectured in 31 and [33]).

In Section 5 we introduce a multipoint (Dirichlet-type) eigenvalue problem which is the analogue of the Dirichlet problem for the Hill equation. We denote by $\left\{\mu_{n}\right\}_{n \in \mathbb{Z}}$ the eigenvalues of this multipoint problem and show that $\left\{\mu_{n}\right\}_{n \in \mathbb{Z}}$ is also characterized as the set of values of $\lambda$ for which there is a proper Floquet solution $f(x ; \lambda)$ such that $f(0 ; \lambda)=0$. If we normalize $f$ so that $f(0 ; \lambda)=1$, then $\left\{\mu_{n}\right\}_{n \in \mathbb{Z}}$ is the set of poles of $f(x ; \lambda)$ (viewed as a function of $\lambda$, of course) counting multiplicities (this approach is used in [11).

We also show (Theorem 7 ) that each gap of the $L^{2}(\mathbb{R})$-spectrum contains exactly one $\mu_{n}$ and each $\psi$-gap of the pseudospectrum contains exactly two $\mu_{n}$ 's, counting multiplicities. Here when we say "gap" or " $\psi$-gap" we also include the endpoints (so that when two consecutive bands or $\psi$-bands touch, the in-between collapsed gap, or $\psi$-gap, is a point). We believe that $\left\{\mu_{n}\right\}_{n \in \mathbb{Z}}$ can be used to formulate the associated inverse spectral problem.

As an application of Theorem 7, we show that if $\nu^{*}$ is a collapsed ("closed") $\psi$-gap, then the Floquet matrix $T\left(\nu^{*}\right)$ is diagonalizable (this too was conjectured in 31] and 33]).

Some of the above results were conjectured in our previous works. However, the formulation of the inverse problem (and, in particular, our conjecture that if all the $\psi$-gaps are closed, then the beam operator is the square of a second-order, Hill-type, operator) remains open.

\section{REVIEW OF EARLIER RESUlts}

2.1. The spectrum. We start by recalling certain general facts related to (1) (see [13, Sec. XIII.7) and some of the main results established in [31] and 33] (other references for Floquet or periodic spectral theory are, e.g., [9, [10, 16], 18, 19, 22], 34]). The problem is selfadjoint (with no boundary conditions at $\pm \infty$ ). The underlying operator $L$ (the "Euler-Bernoulli operator" or "beam operator") is given by

$$
L u=\rho^{-1}\left(a u^{\prime \prime}\right)^{\prime \prime} .
$$

The corresponding Hilbert space is the $\rho$-weighted space $L_{\rho}^{2}(\mathbb{R})$. Notice that $L$ is a product of two second-order differential operators, namely $L=L_{2} L_{1}$, where $L_{1} u=-a u^{\prime \prime}$ and $L_{2} u=-\rho^{-1} u^{\prime \prime}$.

For any fixed $\lambda$ the "shift" transformation

$$
(T u)(x)=u(x+b)
$$

maps solutions of (1) to solutions. As a basis of this space we take the solutions $u_{j}(x ; \lambda), j=1,2,3,4$, such that (primes refer to derivatives with respect to $x ; \delta_{j k}$ is the Kronecker delta)

$$
u_{j}^{(k-1)}(0 ; \lambda)=\delta_{j k}, \quad k=1,2, \quad a(0) u_{j}^{\prime \prime}(0 ; \lambda)=\delta_{j 3}, \quad\left[a u_{j}^{\prime \prime}\right]^{\prime}(0 ; \lambda)=\delta_{j 4} .
$$


We refer to $u_{j}$ as the $j$-th fundamental solution. Each $u_{j}(x ; \lambda)$ is entire in $\lambda$ of order $1 / 4$. We identify $T$ with its matrix representation with respect to the above basis (called Floquet matrix), namely

$$
T=\left[\begin{array}{cccc}
u_{1}(b) & u_{2}(b) & u_{3}(b) & u_{4}(b) \\
u_{1}^{\prime}(b) & u_{2}^{\prime}(b) & u_{3}^{\prime}(b) & u_{4}^{\prime}(b) \\
a(b) u_{1}^{\prime \prime}(b) & a(b) u_{2}^{\prime \prime}(b) & a(b) u_{3}^{\prime \prime}(b) & a(b) u_{4}^{\prime \prime}(b) \\
{\left[a u_{1}^{\prime \prime}\right]^{\prime}(b)} & {\left[a u_{2}^{\prime \prime}\right]^{\prime}(b)} & {\left[a u_{3}^{\prime \prime}\right]^{\prime}(b)} & {\left[a u_{4}^{\prime \prime}\right]^{\prime}(b)}
\end{array}\right]
$$

where the dependence in $\lambda$ is suppressed for typographical convenience. In [31] it was shown that the eigenvalues $r_{1}, r_{2}, r_{3}, r_{4}$ of $T$ (called Floquet multipliers) appear in pairs of inverses, namely

$$
r_{1} r_{4}=r_{2} r_{3}=1
$$

(in fact this is true for any selfadjoint ordinary differential operator with real, periodic coefficients). It follows that the characteristic equation of $T$ has the form

$$
r^{4}-A(\lambda) r^{3}+\widetilde{B}(\lambda) r^{2}-A(\lambda) r+1=0 .
$$

Except for a discrete set of $\lambda$ 's, $T=T(\lambda)$ is similar to a diagonal matrix and its eigenvectors correspond to the Floquet solutions, namely to the solutions $f_{j}$, $j=1,2,3,4$ of (1) such that

$$
f_{j}(x+b)=r_{j} f_{j}(x) .
$$

There are four linearly independent Floquet solutions if and only if $T$ is similar to a diagonal matrix. We also notice that (5) implies

$$
f_{j}(x)=e^{w_{j} x} p_{j}(x), \quad \text { where } \quad r_{j}=e^{w_{j} b} \quad \text { and } \quad p_{j}(x+b)=p_{j}(x) .
$$

The $L_{\rho}^{2}(\mathbb{R})$-spectrum $S(a, \rho)$ of (1) can be characterized as the set

$$
S(a, \rho)=\left\{\lambda \in \mathbb{C}:\left|r_{j}(\lambda)\right|=1, \quad \text { for some } j\right\} .
$$

It can be shown that $S(a, \rho)$ is real with $\inf S(a, \rho)=0$. In the unperturbed case, i.e., when $a(x) \equiv \rho(x) \equiv 1$, we have

$$
S_{0} \stackrel{\text { def }}{=} S(1,1)=[0, \infty)
$$

(the index 0 indicates that a quantity belongs to the unperturbed case).

Next, for a fixed real number $k$, we consider the corresponding $k$-Floquet eigenvalue problem on $(0, b)$, namely

$$
\left[a(x) u^{\prime \prime}(x)\right]^{\prime \prime}=\lambda \rho(x) u(x), \quad u^{(j)}(b)=e^{i k b} u^{(j)}(0), \quad j=0,1,2,3 .
$$

Let $\left\{\lambda_{n}(k)\right\}_{n=1}^{\infty}$ be the spectrum of (7). Since the problem is selfadjoint, $\lambda_{n}(k) \in$ $\mathbb{R}$. The eigenvalues can be indexed so that $\lambda_{n}(k) \leq \lambda_{n+1}(k)$. Then $\lambda_{n}(k) \sim$ $C n^{4}$. We also notice that, since $\lambda_{n}(k+2 \pi / b)=\lambda_{n}(k)$, one only needs to consider $k$ in $[0,2 \pi / b)$. Furthermore, (3) implies that $\lambda_{n}(2 \pi / b-k)=\lambda_{n}(k)$. The set $\left\{\lambda_{n-1} \stackrel{\text { def }}{=} \lambda_{n}(0)\right\}_{n=1}^{\infty}$ is the periodic spectrum, while $\left\{\lambda_{n}^{\prime} \stackrel{\text { def }}{=} \lambda_{n}(\pi / b)\right\}_{n=1}^{\infty}$ is the antiperiodic spectrum. The $n$-th band of $S(a, \rho)$ is the closed interval

$$
B_{n}=\bigcup_{0 \leq k \leq \pi / b} \lambda_{n}(k)
$$


and it is well known that $S(a, \rho)=\bigcup_{n=1}^{\infty} B_{n}$. In [31] it was shown that (as in the Hill equation)

$$
B_{2 m+1}=\left[\lambda_{2 m}, \lambda_{2 m+1}^{\prime}\right], \quad B_{2 m+2}=\left[\lambda_{2 m+2}^{\prime}, \lambda_{2 m+1}\right], \quad m=0,1,2, \ldots .
$$

In fact, as $\lambda$ moves, say with constant velocity, from $\lambda_{2 m}$ to $\lambda_{2 m+1}^{\prime}$ (resp. from $\lambda_{2 m+2}^{\prime}$ to $\lambda_{2 m+1}$ ) two Floquet multipliers, say $r_{2}$ and $r_{3}=\overline{r_{2}}$, move smoothly on the unit circle, with nonvanishing speed (i.e., without changing direction), starting at 1 and arriving at -1 (resp. starting at -1 and arriving at 1 ). The other two multipliers, $r_{1}$ and $r_{4}$, stay real. In particular, $\lambda_{n}(k)$ is strictly monotone in $k$, on $[0, \pi / b]$; hence the interior of $B_{n}$ is never empty. An interesting question here (pointed out by the anonymous referee) is whether we always have $\left|d \lambda_{n} / d k\right|>1$, as in the Hill case (see [19]).

Two bands can "touch" each other (when $\lambda_{2 m+1}=\lambda_{2 m+2}$ or $\lambda_{2 m+1}^{\prime}=\lambda_{2 m+2}^{\prime}$ ), but they cannot overlap (i.e., they have disjoint interiors). The gaps of the spectrum $S(a, \rho)$ are

$$
I_{2 m-1}=\left(\lambda_{2 m-1}^{\prime}, \lambda_{2 m}^{\prime}\right), \quad I_{2 m}=\left(\lambda_{2 m-1}, \lambda_{2 m}\right), \quad m=1,2,3, \ldots,
$$

and empty gaps are traditionally called "closed". If $\lambda_{2 m-1}<\lambda_{2 m}$ (resp. $\lambda_{2 m-1}^{\prime}<$ $\lambda_{2 m}^{\prime}$ ), then $r_{2}$ (and $r_{3}$ ) has square root branch points at $\lambda=\lambda_{2 m-1}, \lambda_{2 m}$ (resp. at $\left.\lambda=\lambda_{2 m-1}^{\prime}, \lambda_{2 m}^{\prime}\right)$. If, on the other hand, $\lambda_{2 m-1}=\lambda_{2 m}$ (resp. $\left.\lambda_{2 m-1}^{\prime}=\lambda_{2 m}^{\prime}\right)$, i.e., if the corresponding gap is closed, then $r_{2}$ (and $r_{3}$ ) are analytic about $\lambda=\lambda_{2 m-1}$ (resp. about $\lambda=\lambda_{2 m-1}^{\prime}$ ). The value $\lambda=\lambda_{0}=0$ is very special since all Floquet multipliers have a fourth root branch point there and $T$ has only one eigenvector.

If $\lambda \neq 0$, then the characteristic equation of $T$ can only have simple or double roots. Now let $\lambda \neq 0$ be such that (4) has a double root, say $r_{j}$. Then there is one Floquet solution $f_{j}(x+b)=r_{j} f_{j}(x)$ and a solution $g_{j}(x)\left(f_{j}\right.$ and $g_{j}$ are linearly independent) such that $g_{j}(x+b)=r_{j} g_{j}(x)+c_{j} f_{j}(x)$, where the constant $c_{j}$ may be 0 (in this case we say that we have coexistence, i.e., two linearly independent Floquet solutions corresponding to the same multiplier). If $c_{j} \neq 0$, we can say that, for this particular $\lambda, T$ has a Jordan anomaly (this terminology is due to Professor Barry Simon) and that $g_{j}(x)$ is a generalized Floquet solution of (11).

We now review the main results of 33 . Notice that, in that reference it was assumed that $a, \rho \in C^{4}(\mathbb{R})$, but we believe that they remain true for $a \in C^{2}(\mathbb{R})$ and $\rho \in C(\mathbb{R})$, and here is why: All these results concern entire functions of $\lambda$ that are polynomial expressions of the $u_{j}(b ; \lambda)$ 's. By considering $a(x)$ and $\rho(x)$ as limits of smooth $\left(C^{4}\right.$ or even $\left.C^{\infty}\right)$ functions $a_{n}(x), \rho_{n}(x)$ in the $C^{2}$ - and sup-norms respectively, the corresponding entire function $u_{j, n}(b ; \lambda)$ converges to $u_{j}(b ; \lambda)$, uniformly on compact subsets of $\mathbb{C}$ (see the proposition in the Appendix), for $j=1,2,3,4$. Thus, we think that our results extend immediately to less smooth coefficients.

For a fixed real number $k$, equation (4) implies (by setting $r=e^{i k b}$ ) that the $k$-Floquet eigenvalues of (7) are the zeros of the entire function

$$
F(\lambda ; k)=B(\lambda)-2 A(\lambda) \cos (k b)+4 \cos ^{2}(k b),
$$

where we have set

$$
B(\lambda)=\widetilde{B}(\lambda)-2 .
$$

In the unperturbed case, this function becomes

$$
F_{0}(\lambda ; k)=4\left[\cosh \left(\lambda^{1 / 4} b\right)-\cos (k b)\right]\left[\cos \left(\lambda^{1 / 4} b\right)-\cos (k b)\right] .
$$


This expression implies easily that, if $0<k<\pi / b$, then the zeros of $F_{0}(\lambda ; k)$ are

$$
\lambda_{n, 0}=\left[2\lfloor n / 2\rfloor \pi / b-(-1)^{n} k\right]^{4}, \quad n=1,2,3, \ldots
$$

(where $\lfloor\cdot\rfloor$ is the greatest integer function) and they are all simple. Furthermore, $\lambda_{n, 0}$ lies in the interior of the $n$-th band $B_{n, 0}$, for every $n$.

(Theorems that have been proved in previous articles are numbered by uppercase Roman letters.)

Theorem A. Let $0<k<\pi / b$. Then the zeros $\left\{\lambda_{n}(k)\right\}_{n=1}^{\infty}$ of $F(\lambda ; k)$ are simple, $\lambda_{n}(k) \in B_{n}$, and to each $\lambda_{n}(k)$ corresponds a unique eigenfunction $\phi_{n}(x ; k)$ of (7), i.e., the geometric multiplicity of $\lambda_{n}(k)$ is also 1 .

Theorem B. The multiplicity of any zero of $F^{+}(\lambda) \stackrel{\text { def }}{=} F(\lambda ; 0)$ can be only 1 or 2. A zero $\lambda^{*}$ of $F^{+}(\lambda)$ is double if and only if $\lambda^{*}=\lambda_{2 m-1}=\lambda_{2 m}$, for some $m \geq 1$ (i.e., the corresponding gap is closed). Furthermore, (7) has two (linearly independent) periodic eigenfunctions corresponding to $\lambda^{*}$ (coexistence) if and only if $\lambda^{*}=\lambda_{2 m-1}=\lambda_{2 m}$. Thus, we can say that the algebraic multiplicity of any periodic eigenvalue is equal to its geometric multiplicity. The same things also hold for $F^{-}(\lambda) \stackrel{\text { def }}{=} F(\lambda ; \pi / b)$, which is the entire function associated to the antiperiodic case.

2.2. The pseudospectrum. The previous results are the exact analogues of the results for the Hill equation regarding algebraic and geometric multiplicities (see [16]).

In [33] we introduced a concept that, as far as we know, does not have a counterpart in the second-order case:

Definition. Let $k \in(0, \pi / b)$. The set

$$
\begin{aligned}
& \Psi_{k}(a, \rho)=\left\{\lambda \in \mathbb{C}: \text { there are two Floquet multipliers } r_{j}(\lambda), r_{l}(\lambda)\right. \text { of (1) } \\
& \text { such that } \left.r_{j}=\bar{r}_{l}=\left|r_{j}\right| e^{i k b}, \quad\left|r_{j}\right|>1\right\}
\end{aligned}
$$

is called the $k$-Floquet pseudospectrum (or $k$-Floquet $\psi$-spectrum) of (1). We, furthermore, call the set

$$
\Psi(a, \rho)=\overline{\bigcup_{0<k<\pi / b} \Psi_{k}(a, \rho)}
$$

the pseudospectrum ( $\psi$-spectrum) of (1) on the line (here $\bar{D}$ denotes the topological closure of $D)$.

The following entire function was introduced in [33]:

$$
G(\lambda ; k)=A(\lambda)^{2}-4 B(\lambda) \cos ^{2}(k b)-16 \cos ^{2}(k b) \sin ^{2}(k b) .
$$

It follows that, if $\nu \in \Psi_{k}(a, \rho)$, then $G(\nu ; k)=0$.

Since

$$
G(\lambda ; k)=G(\lambda ; \pi / b-k),
$$

the zeros of $G(\lambda ; k)$ also include $\Psi_{\pi / b-k}(a, \rho)$.

Conversely, for a fixed $k \in(0, \pi / 2 b)$, let $\nu$ be a zero of $G(\lambda ; k)$. Then (12) implies that

$$
A(\nu)^{2}-4 B(\nu) \cos ^{2}(k b)-16 \cos ^{2}(k b) \sin ^{2}(k b)=0 .
$$


Now by (3), (4), and (9),

$$
A=r_{1}+\frac{1}{r_{1}}+r_{2}+\frac{1}{r_{2}} \quad \text { and } \quad B=\left(r_{1}+\frac{1}{r_{1}}\right)\left(r_{2}+\frac{1}{r_{2}}\right) ;
$$

hence, given $r_{1}$, (13) becomes a 4-th degree (algebraic) equation in $r_{2}$. One can check that its solutions are

$$
r_{2}=r_{1} e^{ \pm 2 i k b} \quad \text { and } \quad r_{2}=r_{1}^{-1} e^{ \pm 2 i k b} .
$$

This means that

$$
\nu \in \Psi_{k}(a, \rho) \cup \Psi_{\pi / b-k}(a, \rho)=\left\{\lambda: r_{j}=r_{l} e^{ \pm 2 i k b}, \quad\left|r_{j}\right| \neq 1\right\} .
$$

Therefore, for $k \in(0, \pi / b), k \neq \pi /(2 b)$, the set of zeros of $G(\lambda ; k)$ is $\Psi_{k}(a, \rho) \cup$ $\Psi_{\pi / b-k}(a, \rho)$.

The value $k=\pi /(2 b)$ is somehow special since

$$
G(\lambda ; \pi /(2 b))=A(\lambda)^{2} .
$$

It follows that, if $\nu$ is a zero of $G(\lambda ; \pi /(2 b))$, then

$$
r_{2}(\nu)=-r_{1}(\nu) \quad \text { or } \quad r_{2}=-r_{1}(\nu)^{-1} .
$$

Hence

$$
\nu \in \Psi_{\pi / 2 b}(a, \rho)=\left\{\lambda: r_{j}=-r_{l}, \quad\left|r_{j}\right| \neq 1\right\}
$$

(notice that, in this case all Floquet multipliers are pure imaginary). These results are implicitly contained in [33], where the following theorem was established:

Theorem C. Let $0<k<\pi / b$. If $k \neq \pi /(2 b)$ the zeros of the entire function $G(\lambda ; k)$, defined in (12), are all real, strictly negative, and simple. The zeros of $G(\lambda ; \pi /(2 b))$ are all real strictly negative and double. Each zero $\nu_{n}(k)$ of $G(\lambda ; k)$ is (as a function of $k$ ) strictly monotone on the interval $(0, \pi /(2 b))$ and on $(\pi /(2 b), \pi / b)$.

The function $G(\lambda ; k)$, as defined in (12), makes sense for all $k \in \mathbb{C}$ (in fact, it is entire in $\lambda, k)$. In particular,

$$
E(\lambda) \stackrel{\text { def }}{=} G(\lambda ; 0)=A(\lambda)^{2}-4 B(\lambda) .
$$

This function was introduced in 31. It was shown there that 0 is always a simple zero of $E(\lambda)$ and that $\nu \neq 0$ is a zero of $E(\lambda)$ if and only if $r_{j}(\nu)=r_{l}(\nu) \neq \pm 1$, $j \neq l$. In [33] the following theorem was proved:

Theorem D. The nonzero zeros of $E(\lambda)$ are all real, strictly negative, and simple or double. If we denote them by

$$
0=\nu_{0}>\nu_{1}^{\prime} \geq \nu_{2}^{\prime}>\nu_{1} \geq \nu_{2}>\nu_{3}^{\prime} \geq \nu_{4}^{\prime}>\cdots,
$$

we have a pseudoband-pseudogap structure on the negative $\lambda$-axis. Each $\psi$-band $\left[\nu_{0}, \nu_{1}^{\prime}\right],\left[\nu_{2}^{\prime}, \nu_{1}\right],\left[\nu_{2}, \nu_{3}^{\prime}\right], \ldots$ contains exactly one point of the $\psi$-spectrum $\Psi_{k}(a, \rho)$, for any fixed $k \in(0, \pi / b)$.

Remark 1 . Since 0 is always a simple zero of $E(\lambda)$ and in the unperturbed case we have

$$
E_{0}(\lambda)=4\left[\cosh \left(\lambda^{1 / 4} b\right)-\cos \left(\lambda^{1 / 4} b\right)\right]^{2},
$$

it follows by Theorem D and a simple continuity argument that $E(\lambda)>0$, if $\lambda>0$ or if $\lambda$ is in the interior of a $\psi$-gap; whereas $E(\lambda)<0$, if $\lambda$ is in the interior of a 
$\psi$-band. At the zeros $\nu_{n}, \nu_{n}^{\prime}, n \neq 0$, of $E(\lambda)$, there are Floquet multipliers $r_{j}, r_{l}$, $j \neq l$, such that $r_{j}\left(\nu_{n}\right)=r_{l}\left(\nu_{n}\right)>1$ and $r_{j}\left(\nu_{n}^{\prime}\right)=r_{l}\left(\nu_{n}^{\prime}\right)<-1$.

If $a(x) \rho(x) \equiv 1$ (in this case the beam operator is a "perfect square"), then all the nonzero zeros of $E(\lambda)$ are double, i.e., $\lambda E(\lambda)$ is the square of an entire function. Equivalently, all $\psi$-gaps are closed, i.e., empty.

For the unperturbed case we have

$$
G_{0}(\lambda ; k)=4\left\{\cos \left[\lambda^{1 / 4} b(1+i)\right]-\cos 2 k b\right\}\left\{\cos \left[\lambda^{1 / 4} b(1-i)\right]-\cos 2 k b\right\} .
$$

The zeros of $G_{0}(\lambda ; k)$ are $\nu_{n, 0}(k)$ and $\nu_{n, 0}(\pi / b-k), n=1,2,3, \ldots$, where

$$
\nu_{n, 0}(k)=-4\left[2\lfloor n / 2\rfloor \pi / b-(-1)^{n} k\right]^{4} .
$$

Next we set

$$
\nu_{n-1,0}=\nu_{n, 0}(0)=\lim _{k \searrow 0} \nu_{n, 0}(k), \quad \nu_{n, 0}^{\prime}=\nu_{n, 0}(\pi / b)=\lim _{k \nearrow \pi / b} \nu_{n, 0}(k) .
$$

Thus, $\nu_{0,0}=0$ and, for $m=1,2,3, \ldots$,

$$
\begin{aligned}
& \nu_{2 m-1,0}=\nu_{2 m, 0}=-4\left(\frac{2 m \pi}{b}\right)^{4}, \\
& \nu_{2 m-1,0}^{\prime}=\nu_{2 m, 0}^{\prime}=-4\left[\frac{(2 m-1) \pi}{b}\right]^{4} .
\end{aligned}
$$

These numbers are the zeros of $E_{0}(\lambda)$.

2.2.1. The significance of the pseudospectrum. The purpose of this short (sub)subsection is to elucidate certain things regarding the concept of the pseudospectrum.

Let $L$ be an $n$-th order (ordinary) differential operator with periodic coefficients. Then one can consider the Floquet multipliers $r_{j}(\lambda), j=1, \ldots, n$, of $L$ and the corresponding Floquet solutions $f_{j}(x ; \lambda), j=1, \ldots, n$. The $r_{j}(\lambda)$ 's are, in fact, the branches of a (multivalued) analytic function which we denote by $r(\lambda)$ and, similarly, the $f_{j}(x ; \lambda)$ 's are the branches of a $\lambda$-analytic function $f(x ; \lambda)$.

Let $\Gamma$ be the Riemann surface of $r(\lambda)$. If we normalize $f(x ; \lambda)$ so that $f(0 ; \lambda)=1$, then $f(x ; \lambda)$ becomes a meromorphic function on $\Gamma$, whose set of poles we denote by $\left\{\mu_{n}\right\}$.

In $[1]$ it is suggested that the (periodic) inverse spectral data for $L$ is the Riemann surface $\Gamma$ together with the set of poles $\left\{\mu_{n}\right\}$ (notice that each $\mu_{n}$ is a point on $\Gamma$, i.e., $\mu_{n}$ is not just a complex number, since it also contains the information: on which sheet of $\Gamma$ the pole lies). This is, of course, inspired by the inverse theory of the Hill operator (see, e.g., [11, [14, [24], [25], 38]).

If $L$ is the Euler-Bernoulli operator, the multiplier $r(\lambda)$ has two types of branch points (the point $\lambda=0$ is special and can be considered as being of both types). The branch points of the first type lie on the positive real axis and are the endpoints of the bands of the $L_{\rho}^{2}(\mathbb{R})$-spectrum (they are also the periodic and antiperiodic eigenvalues), exactly as in the Hill case. The branch points of the second type lie on the negative real axis and they, too, define a band-gap structure which we have called pseudospectrum (we have called its bands and gaps " $\psi$-bands" and " $\psi$-gaps" respectively to distinguish them from the spectral bands and gaps).

Each gap of the spectrum contains exactly one $\mu_{n}$. But now there are $\mu_{n}$ 's that do not lie in any spectral gap. It turns out that each $\psi$-gap of the pseudospectrum 
contains exactly two of those $\mu_{n}$ 's, counting multiplicities. The exact statement is Theorem 7 of Section 5, which is, perhaps, the crux of this work.

In conclusion, for the Euler-Bernoulli case, we need both the $L_{\rho}^{2}(\mathbb{R})$-spectrum and the pseudospectrum in order to determine the Riemann surface $\Gamma$ and the intervals in which the $\mu_{n}$ 's are confined. This is why we believe that the pseudospectrum plays an essential role in the Euler-Bernoulli inverse spectral theory. In particular, we have conjectured that if the pseudospectrum has no gaps (i.e., if it is the interval $(-\infty, 0])$, then the Euler-Bernoulli operator is a perfect square of a Hill-type operator.

\section{The zeros of the Function $F(\lambda ; k)$, FOR COMPlex $k$}

3.1. The technique. There is a technique that we have employed for proving some of our statements regarding (1), especially properties of quantities that depend on (or are related to) the spectral parameter $\lambda$ (e.g., this technique has been already used for proving Theorems $\mathrm{C}$ and $\mathrm{D}$ mentioned above). It combines continuity arguments and large $|\lambda|$ asymptotics.

Here is how the technique works: We first check that the property we want to establish holds in the unperturbed case $a(x) \equiv \rho(x) \equiv 1$. Then we deform $a(x)$ and $\rho(x)$ continuously until we reach the general case, making sure that the property remains valid (a kind of "continuous induction"). For example, we can specify the (obviously continuous) deformation

$$
a(x ; t)=t a(x)+(1-t), \quad \rho(x ; t)=c(t)^{4}[t \rho(x)+(1-t)], \quad t \in[0,1],
$$

where

Notice that

$$
c(t)=\frac{b}{\int_{0}^{b}\left[\frac{t \rho(x)+(1-t)}{t a(x)+(1-t)}\right]^{1 / 4} d x} .
$$

$$
a(x ; 0) \equiv \rho(x ; 0) \equiv 1, \quad a(x ; 1)=a(x) \quad \text { and } \quad \rho(x ; 1)=\rho(x) .
$$

Also, since $a(x)$ and $\rho(x)$ are strictly positive, it follows that there is a constant $\delta_{0}$, independent of $t$, such that

$$
a(x ; t), \rho(x ; t) \geq \delta_{0}>0, \quad \text { for all } t \in[0,1],
$$

where $a(x ; t)$ and $\rho(x ; t)$ satisfy the normalization condition (2), namely

$$
\int_{0}^{b}\left[\frac{\rho(x ; t)}{a(x ; t)}\right]^{1 / 4} d x=b, \quad \text { for all } t \in[0,1] .
$$

Finally, as functions of $x, a(x ; t)$ and $\rho(x ; t)$ are $b$-periodic and as smooth as $a(x)$ and $\rho(x)$ respectively.

In some cases, we first prove the desired result for $a(x)$ and $\rho(x)$ that are sufficiently smooth, say $a, \rho \in C^{4}(\mathbb{R})$, and then extend it to the more general case $a \in C^{2}(\mathbb{R})$ and $\rho \in C(\mathbb{R})$ by approximating $a(x)$ and $\rho(x)$ by smooth functions (in the $C^{2}$ - and sup-norms respectively). The fact that a $\lambda$-quantity of the smooth case approaches (uniformly on compact subsets of $\mathbb{C}$ ) the corresponding $\lambda$-quantity of the more general case usually follows from standard Gronwall-type estimates (e.g., see the proposition in the Appendix). 
One main reason that we need smooth $a(x)$ and $\rho(x)$ is that, if this is the case, there is a Liouville-type transformation (found in [3]) that transforms (11) to a canonical fourth-order eigenvalue equation, namely

$$
v^{\prime \prime \prime \prime}(\xi)-\left[q_{1}(\xi) v^{\prime}(\xi)\right]^{\prime}+q_{2}(\xi) v(\xi)=\lambda v(\xi),
$$

where

$$
\xi=\int_{0}^{x}\left[\frac{\rho(y)}{a(y)}\right]^{1 / 4} d y, \quad v(\xi)=\rho(x)^{3 / 8} a(x)^{1 / 8} u(x)
$$

and $q_{1}(\xi), q_{2}(\xi)$ are $b$-periodic expressions involving $a$ and $\rho$ (see [3]).

Then one can use in (20) the asymptotic estimates of [29], Part I, Chapt. II, to conclude that, in each sector

$$
S_{l}=\left\{\lambda \in \mathbb{C}: \frac{l \pi}{4} \leq \arg (\lambda) \leq \frac{(l+1) \pi}{4}\right\}, \quad l=0,1, \ldots, 7,
$$

of the complex $\lambda$-plane there are four $\lambda$-analytic linearly independent solutions $\phi_{j}(x ; t ; \lambda), j=1,2,3,4$, of

$$
\left[a(x ; t) u^{\prime \prime}(x)\right]^{\prime \prime}=\lambda \rho(x ; t) u(x),
$$

such that, given $M>0$,

$$
\left|\phi_{j}^{\prime \prime}(x ; t ; \lambda)-\frac{\rho(x ; t)^{1 / 8}}{a(x ; t)^{5 / 8}} \varepsilon_{j}^{2} \lambda^{1 / 2} e^{\varepsilon_{j} \lambda^{1 / 4} S(x ; t)}\right| \leq K\left|\lambda^{1 / 4} e^{\varepsilon_{j} \lambda^{1 / 4} S(x ; t)}\right|, \quad 0 \leq x \leq M,
$$

$$
\left|\phi_{j}^{\prime \prime \prime}(x ; t ; \lambda)-\frac{\rho(x ; t)^{3 / 8}}{a(x ; t)^{7 / 8}} \varepsilon_{j}^{3} \lambda^{3 / 4} e^{\varepsilon_{j} \lambda^{1 / 4} S(x ; t)}\right| \leq K\left|\lambda^{1 / 2} e^{\varepsilon_{j} \lambda^{1 / 4} S(x ; t)}\right|, \quad 0 \leq x \leq M,
$$

where

$$
S(x ; t)=\int_{0}^{x}\left[\frac{\rho(y ; t)}{a(y ; t)}\right]^{1 / 4} d y
$$

(in particular, $S(n b ; t)=n b$, if $n \in \mathbb{Z})$. Here $\lambda^{1 / 4}$ stands for the principal branch of the fourth root (so that $\Re\left\{\lambda^{1 / 4}\right\} \geq 0, \Im\left\{\lambda^{1 / 4}\right\} \geq 0$ ), $\left\{\varepsilon_{1}, \varepsilon_{2}, \varepsilon_{3}, \varepsilon_{4}\right\}=\{i,-1,-i, 1\}$, and the (positive) constant $K$ depends on $a(x), \rho(x)$, and $M$, but not on $t$.

We finish this subsection with a useful lemma.

Lemma 1. If $r_{j}(\lambda ; t), j=1,2,3,4$, are the Floquet multipliers of (21), where $t \in[0,1]$, then

$$
\left|\frac{r_{j}(\lambda ; t)}{e^{\varepsilon_{j} \lambda^{1 / 4} b}}-1\right| \leq \frac{K}{|\lambda|^{1 / 4}}, \quad j=1,2,3,4,
$$

where $\lambda^{1 / 4}$ is the principal branch of the fourth root, $\left\{\varepsilon_{1}, \varepsilon_{2}, \varepsilon_{3}, \varepsilon_{4}\right\}=\{i,-1,-i, 1\}$, and the constant $K>0$ depends on $a(x), \rho(x)$, but not on $t$. 
Proof. Let

$$
T=\left[T_{j k}\right]_{1 \leq j, k \leq 4}
$$

be the Floquet matrix of (21) with respect to the basis $\phi_{j}(x ; t ; \lambda), j=1,2,3,4$, where $\phi_{j}(x ; t ; \lambda)$ is the solution of (21) that satisfies (22), (23), (24), and (25). It follows that, as $|\lambda| \rightarrow \infty$,

$$
T_{j k}=e^{\varepsilon_{k} \lambda^{1 / 4} b}\left[\delta_{j k}+O\left(\lambda^{-1 / 4}\right)\right], \quad \text { uniformly in } t,
$$

where $\delta_{j k}$ is the Kronecker delta. As we know, $r_{j}(\lambda ; t), j=1,2,3,4$, are the roots of the equation

$$
r^{4}-A(\lambda ; t) r^{3}+[B(\lambda ; t)+2] r^{2}-A(\lambda ; t) r+1=0,
$$

where

$$
A(\lambda ; t)=\sum_{j=1}^{4} T_{j j}
$$

and

$$
B(\lambda ; t)+2=\sum_{1 \leq j<k \leq 4}\left|\begin{array}{cc}
T_{j j} & T_{j k} \\
T_{k j} & T_{k k}
\end{array}\right| .
$$

Thus (27) implies that, as $|\lambda| \rightarrow \infty$,

$$
A(\lambda ; t)=e^{\lambda^{1 / 4} b}\left[1+O\left(\lambda^{-1 / 4}\right)\right]+e^{-i \lambda^{1 / 4} b}\left[1+O\left(\lambda^{-1 / 4}\right)\right]
$$

and

$$
\begin{aligned}
B(\lambda ; t)+2= & e^{\lambda^{1 / 4} b} e^{-i \lambda^{1 / 4} b}\left[1+O\left(\lambda^{-1 / 4}\right)\right]+e^{\lambda^{1 / 4} b} e^{i \lambda^{1 / 4} b}\left[1+O\left(\lambda^{-1 / 4}\right)\right] \\
& +e^{-\lambda^{1 / 4} b} e^{-i \lambda^{1 / 4} b}\left[1+O\left(\lambda^{-1 / 4}\right)\right]+e^{-\lambda^{1 / 4} b} e^{-i \lambda^{1 / 4} b}\left[1+O\left(\lambda^{-1 / 4}\right)\right]
\end{aligned}
$$

uniformly in $t$. The statement of the lemma follows easily from these two formulas.

3.2. The theorems. The analysis that follows is inspired by the work [20] of W. Kohn for the second-order case (see also 1]). Consider again the function of formula (8), namely

$$
F(\lambda ; k)=B(\lambda)-2 A(\lambda) \cos (k b)+4 \cos ^{2}(k b) .
$$

This function is the analogue of the function

$$
\Delta(\lambda)-2 \cos (k b)
$$

that appears in the analysis of the Hill operator (see, e.g., 1]). Notice that, although $F(\lambda ; k)$ is usually viewed as an entire function of $\lambda$, it is entire in both $\lambda$ and $k$. We want to generalize Theorems A and $\mathrm{B}$ for the case of complex $k$.

Theorem 1. For a fixed $k \in \mathbb{C}$, let $\lambda^{*}$ be a zero of $F(\lambda ; k)$ of multiplicity $m\left(\lambda^{*}\right)>1$. Then $\lambda^{*}$ is also a zero of the entire function

$$
D(\lambda)=E^{\prime}(\lambda)^{2}-4 A^{\prime}(\lambda)^{2} E(\lambda) .
$$

Furthermore, $D(\lambda) \not \equiv 0$. 
Proof. The derivative of $F(\lambda ; k)$ with respect to $\lambda$ satisfies

$$
F_{\lambda}\left(\lambda^{*} ; k\right)=B^{\prime}\left(\lambda^{*}\right)-2 A^{\prime}\left(\lambda^{*}\right) \cos (k b)=0 .
$$

A straightforward calculation (using (16)) can verify that

$$
A^{\prime}(\lambda)^{2} F(\lambda ; k)-A(\lambda) A^{\prime}(\lambda) F_{\lambda}(\lambda ; k)+2 B^{\prime}(\lambda) F_{\lambda}(\lambda ; k)-F_{\lambda}(\lambda ; k)^{2}=\frac{D(\lambda)}{16},
$$

where $D(\lambda)$ is given by (28). Thus, if $F\left(\lambda^{*} ; k\right)=F_{\lambda}\left(\lambda^{*} ; k\right)=0$, then $D\left(\lambda^{*}\right)=0$.

If $D(\lambda) \equiv 0$, then all zeros of $F(\lambda ; k)$ should have been multiple. But, by Theorem $\mathrm{A}$ the zeros of $F(\lambda ; k)$ are simple, if $k$ is real and $0<k<\pi / b$. Thus, $D(\lambda) \not \equiv 0$.

One important feature of Theorem 1 is that $D(\lambda)$ is independent of $k$. The next theorem is quite informative. To prove it we employ the technique described in the previous subsection.

Theorem 2. All the zeros of the entire function $D(\lambda)$ of (28) are real and they are located as follows: (a) $D(\lambda)$ has exactly one (simple) zero in each gap of the spectrum $S(a, \rho)$ of (11) (with the understanding that, if the gap is closed, i.e., collapses to a double periodic or antiperiodic eigenvalue, say $\lambda^{*}$, then the simple zero of $D(\lambda)$ is $\lambda^{*}$ ); (b) $D(\lambda)$ has exactly two zeros (counting multiplicities) in each $\psi$-gap of the pseudospectrum of (11). In case (b), if the $\psi$-gap is open, then $D(\lambda)$ has exactly two simple zeros in it, whereas if the $\psi$-gap is closed, i.e., collapses to a point $\nu^{*}$, where $\nu^{*}=\nu_{2 n-1}=\nu_{2 n}$, or $\nu^{*}=\nu_{2 n-1}^{\prime}=\nu_{2 n}^{\prime}$, for some $n=1,2,3, \ldots$ (see the statement of Theorem $D)$, then $\nu^{*}$ is a double zero of $D(\lambda)$. There are no other zeros of $D(\lambda)$.

Proof. We start by examining the zeros of $D(\lambda)$ in the $\psi$-gaps. Let $(\alpha, \beta)$ be a $\psi$ gap. If it is closed, i.e., if $\alpha=\beta$, then, by Theorem $\mathrm{D}$ we have $E(\alpha)=E^{\prime}(\alpha)=0$. Thus (28) implies that $D(\alpha)=D^{\prime}(\alpha)=0$ (i.e., $\alpha$ is a zero of $D(\lambda)$ of multiplicity $\geq 2$ ). Next assume that $\alpha<\beta$. We then have $E(\alpha)=E(\beta)=0$, while, by Remark $1, E(\lambda)>0$, for $\lambda \in(\alpha, \beta)$. It follows that there is a $\gamma \in(\alpha, \beta)$ such that $E^{\prime}(\gamma)=0$. Then (28) implies that

$$
D(\alpha)=E^{\prime}(\alpha)^{2}>0, \quad D(\beta)=E^{\prime}(\beta)^{2}>0, \quad D(\gamma)=-4 A^{\prime}(\gamma)^{2} E(\gamma)<0 .
$$

Therefore, $D(\lambda)$ must have a zero in $(\alpha, \gamma)$ and one in $(\gamma, \beta)$. In conclusion, $D(\lambda)$ always has at least two zeros (counting multiplicities) in each $\psi$-gap, even if it is closed.

Next we consider the unperturbed case. Using $E_{0}(\lambda)$ of (17), namely

$$
E_{0}(\lambda)=4\left[\cosh \left(\lambda^{1 / 4} b\right)-\cos \left(\lambda^{1 / 4} b\right)\right]^{2},
$$

and the fact that

$$
A_{0}(\lambda)=2\left[\cosh \left(\lambda^{1 / 4} b\right)+\cos \left(\lambda^{1 / 4} b\right)\right]
$$

we get from (28) that

$$
D_{0}(\lambda)=\frac{16 b^{2}}{\lambda^{3 / 2}} \sinh \left(\lambda^{1 / 4} b\right) \sin \left(\lambda^{1 / 4} b\right)\left[\cosh \left(\lambda^{1 / 4} b\right)-\cos \left(\lambda^{1 / 4} b\right)\right]^{2}
$$


(in particular, $D_{0}(0)=16 b^{8} \neq 0$ ). Hence the zeros of $D_{0}(\lambda)$ are exactly as the theorem describes them. As we deform the unperturbed case continuously, until we reach (1), $D(\lambda)$ will continue to have two zeros in each $\psi$-gap, unless some new nonreal zeros enter the $\psi$-gap. Also, by Theorem A and Theorem 1 , the simple zero that $D(\lambda)$ has in each gap of the spectrum $S(a, \rho)$ (including the case of a closed gap) cannot move into the interior of a band. Hence this zero will remain in the gap until some other nonreal zero enters the gap. Therefore, as we deform the unperturbed case, $D(\lambda)$ will continue to have exactly one zero in each gap and exactly two zeros (counting multiplicities) in each $\psi$-gap, until some nonreal zero(s) enters a gap or a $\psi$-gap. But where can these nonreal zeros come from? Since $D_{0}(\lambda)$ has no other zeros, they can only come from infinity.

From the above it follows that, in order to finish the proof, we need to demonstrate that, as we deform the unperturbed case, no new zeros of $D(\lambda)$ can appear from infinity. Assume first that $a, \rho \in C^{\infty}(\mathbb{R})$. Let $D\left(\lambda^{*}\right)=0$ where $\lambda^{*}$ does not satisfy the statement of the theorem. Consider the continuous deformation (19) and let $D(\lambda ; t)$ be the function $D(\lambda)$ for the problem (21). Then there is a $t_{0} \in[0,1)$ and a zero $\lambda_{\omega}(t)$ of $D(\lambda ; t)$ such that

$$
\lambda_{\omega}(1)=\lambda^{*} \quad \text { and } \quad \lim _{t \searrow t_{0}}\left|\lambda_{\omega}(t)\right|=\infty
$$

$\left(\lambda_{\omega}(t)\right.$ depends continuously on $\left.t\right)$.

By Theorem 1 there is a $k=k(t) \in \mathbb{C}$ such that

$$
F\left(\lambda_{\omega}(t) ; k ; t\right)=0
$$

and

$$
F_{\lambda}\left(\lambda_{\omega}(t) ; k ; t\right)=0 .
$$

An equivalent way to state (33) is to say that

$$
r_{j}=r_{j}\left(\lambda_{\omega}(t) ; t\right)=e^{i k b}, \quad \text { for some } \quad j=1,2,3,4 \text {, }
$$

namely

$$
r_{1}+r_{4}=r_{1}+r_{1}^{-1}=2 \cos (k b) \quad \text { or } \quad r_{2}+r_{3}=r_{2}+r_{2}^{-1}=2 \cos (k b) .
$$

Using (29) and (35), formula (34) becomes

$$
B_{\lambda}\left(\lambda_{\omega}(t) ; k ; t\right)=A_{\lambda}\left(\lambda_{\omega}(t) ; k ; t\right)\left[r_{j}\left(\lambda_{\omega}(t) ; t\right)+r_{j}\left(\lambda_{\omega}(t) ; t\right)^{-1}\right], \quad j=1 \text { or } 2 .
$$

By recalling (14), (36) becomes

$$
\begin{aligned}
{\left[r_{2}\left(\lambda_{\omega}\right)\right.} & \left.+r_{2}\left(\lambda_{\omega}\right)^{-1}\right]\left.\partial_{\lambda}\left[r_{1}+r_{1}^{-1}\right]\right|_{\lambda=\lambda_{\omega}} \\
& +\left.\left[r_{1}\left(\lambda_{\omega}\right)+r_{1}\left(\lambda_{\omega}\right)^{-1}\right] \partial_{\lambda}\left[r_{2}+r_{2}^{-1}\right]\right|_{\lambda=\lambda_{\omega}} \\
= & {\left.\left[r_{j}\left(\lambda_{\omega}\right)+r_{j}\left(\lambda_{\omega}\right)^{-1}\right] \partial_{\lambda}\left[r_{1}+r_{1}^{-1}\right]\right|_{\lambda=\lambda_{\omega}} } \\
& +\left.\left[r_{j}\left(\lambda_{\omega}\right)+r_{j}\left(\lambda_{\omega}\right)^{-1}\right] \partial_{\lambda}\left[r_{2}+r_{2}^{-1}\right]\right|_{\lambda=\lambda_{\omega}}
\end{aligned}
$$


where the dependence in $t$ is suppressed for typographical convenience and $j=1$ or 2. Thus,

$$
\begin{aligned}
& {\left.\left[r_{2}\left(\lambda_{\omega}\right)+r_{2}\left(\lambda_{\omega}\right)^{-1}\right] \partial_{\lambda}\left[r_{1}+r_{1}^{-1}\right]\right|_{\lambda=\lambda_{\omega}}} \\
& \quad=\left.\left[r_{1}\left(\lambda_{\omega}\right)+r_{1}\left(\lambda_{\omega}\right)^{-1}\right] \partial_{\lambda}\left[r_{1}+r_{1}^{-1}\right]\right|_{\lambda=\lambda_{\omega}},
\end{aligned}
$$

if $j=1$, or

$$
\begin{aligned}
& {\left.\left[r_{1}\left(\lambda_{\omega}\right)+r_{1}\left(\lambda_{\omega}\right)^{-1}\right] \partial_{\lambda}\left[r_{2}+r_{2}^{-1}\right]\right|_{\lambda=\lambda_{\omega}}} \\
& \quad=\left.\left[r_{2}\left(\lambda_{\omega}\right)+r_{2}\left(\lambda_{\omega}\right)^{-1}\right] \partial_{\lambda}\left[r_{2}+r_{2}^{-1}\right]\right|_{\lambda=\lambda_{\omega}},
\end{aligned}
$$

if $j=2$. Therefore,

$$
r_{1}\left(\lambda_{\omega}\right)+r_{1}\left(\lambda_{\omega}\right)^{-1}=r_{2}\left(\lambda_{\omega}\right)+r_{2}\left(\lambda_{\omega}\right)^{-1}
$$

or

or

$$
\left[1-r_{1}\left(\lambda_{\omega}\right)^{-2}\right] \partial_{\lambda} r_{1}\left(\lambda_{\omega}\right)=0
$$

$$
\left[1-r_{2}\left(\lambda_{\omega}\right)^{-2}\right] \partial_{\lambda} r_{2}\left(\lambda_{\omega}\right)=0
$$

An equivalent way to write the last two equations is

$$
\left[r_{1}\left(\lambda_{\omega}\right)^{2}-1\right]\left[r_{2}\left(\lambda_{\omega}\right)^{2}-1\right]=0
$$

or

$$
\left[\partial_{\lambda} r_{1}\left(\lambda_{\omega}\right)\right]\left[\partial_{\lambda} r_{2}\left(\lambda_{\omega}\right)\right]=0 .
$$

Thus, if $D\left(\lambda_{\omega} ; t\right)=0$, then $\lambda_{\omega}$ satisfies (37), or (38), or (39). But (37) means that $E\left(\lambda_{\omega} ; t\right)=0$ and, by Theorem 1, this can happen if and only if $\lambda_{\omega}$ is a double zero of $E(\lambda ; t)$. Similarly, (38) means that $\lambda_{\omega}$ is a periodic or antiperiodic eigenvalue of (21) and this can happen (see Theorem B) if and only if $\lambda_{\omega}$ is a double such eigenvalue, equivalently if $\lambda_{\omega}$ is a double zero of $F^{+}(\lambda ; t) F^{-}(\lambda ; t)$. Therefore, either $\lambda_{\omega}(t)$ is a double zero of

$$
F^{+}(\lambda ; t) F^{-}(\lambda ; t) E(\lambda ; t),
$$

or $\lambda_{\omega}(t)$ satisfies (39).

Next, let $\left\{z_{-n}(t)\right\}_{n=0}^{\infty}$ be the zeros (counting multiplicities) of $E(\lambda ; t)$ and $\left\{z_{n}(t)\right\}_{n=0}^{\infty}$ be the zeros (counting multiplicities) of $F^{+}(\lambda ; t) F^{-}(\lambda ; t)$ (thus $z_{0}(t)=$ $0)$. Furthermore, we assume that $\left\{z_{n}(t)\right\}_{n=-\infty}^{\infty}$ is increasing in $n$. We then have the estimates (see [33])

$$
\left|z_{n}(t)-z_{n}(0)\right| \leq K n^{2}, \quad \text { for all } n \in \mathbb{Z},
$$

where the (positive) constant $K$ is independent of $t \in[0,1], z_{0}(0)=0$, and, by (11) and (18),

$$
z_{1-2 l}(0)=z_{-2 l}(0)=-4\left(\frac{l \pi}{b}\right)^{4}, \quad z_{2 l-1}(0)=z_{2 l}(0)=\left(\frac{l \pi}{b}\right)^{4}, \quad l=1,2,3, \ldots .
$$

In particular, there is a constant $C>0$ such that

$$
z_{n+2}(0)-z_{n}(0) \geq C|n|^{3}, \quad \text { for all } n \in \mathbb{Z} .
$$

Finally, recall that $z_{n}(t)$ is a branch point of $r(\lambda ; t)$ if and only if $z_{n}(t)$ is a simple zero of $F^{+}(\lambda ; t) F^{-}(\lambda ; t)$ or $E(\lambda ; t)$. 
We will now show that (32) is impossible. This will imply that there is no $\lambda^{*}$, such that $D\left(\lambda^{*}\right)=0$, violating the statement of the theorem. Let $\Gamma=\Gamma\left(\lambda_{\omega}(t), R\right)$, the circle in the complex plane with radius $R$, centered at $\lambda_{\omega}(t)$. We assume that $R$ is small enough so that $\Gamma$ does not enclose any branch point of $r(\lambda ; t)$, i.e., any $\left(z_{n}(t)\right.$ which is a) simple zero of $F^{+}(\lambda ; t) F^{-}(\lambda ; t)$ or $E(\lambda ; t)$. Then by Cauchy's integral formula we have

$$
\partial_{\lambda} r_{j}\left(\lambda_{\omega}\right)-\frac{\varepsilon_{j} b}{4 \lambda_{\omega}^{3 / 4}} e^{\varepsilon_{j} \lambda_{\omega}^{1 / 4} b}=\frac{1}{2 \pi i} \int_{\Gamma} \frac{r_{j}(z)-e^{\varepsilon_{j} z^{1 / 4} b}}{\left(z-\lambda_{\omega}\right)^{2}} d z .
$$

Thus, if $\lambda_{\omega}(t)$ satisfies (39),

$$
\left|\frac{\varepsilon_{j} b}{4 \lambda_{\omega}^{3 / 4}} e^{\varepsilon_{j} \lambda_{\omega}^{1 / 4} b}\right| \leq \frac{1}{2 \pi R} \int_{0}^{2 \pi}\left|r_{j}(z(\theta))-e^{\varepsilon_{j} z(\theta)^{1 / 4} b}\right| d \theta, \quad z(\theta)=\lambda_{\omega}(t)+e^{i \theta} R .
$$

If we assume, in accordance with 32 , that

$$
\left|\lambda_{\omega}(t)\right| \rightarrow \infty
$$

and take

$$
R=\left|\lambda_{\omega}(t)\right|^{(1 / 2)+\varepsilon}, \quad \text { for some } \varepsilon \in(0,1 / 4),
$$

then (43) and (26) imply that there is a constant $K$ independent of $t$ such that

$$
\left|\frac{1}{\lambda_{\omega}(t)^{3 / 4}} e^{\varepsilon_{j} \lambda_{\omega}^{1 / 4} b}\right| \leq \frac{K}{R} \frac{\left|e^{\varepsilon_{j} \lambda_{\omega}^{1 / 4} b}\right|}{\left|\lambda_{\omega}(t)\right|^{1 / 4}}=K \frac{\left|e^{\varepsilon_{j} \lambda_{\omega}^{1 / 4} b}\right|}{\left|\lambda_{\omega}(t)\right|^{(3 / 4)+\varepsilon}} .
$$

This inequality is obviously impossible as $\left|\lambda_{\omega}(t)\right|$ gets arbitrarily large; hence $\Gamma$ must enclose branch points. We have, therefore, established the following: Given $\varepsilon \in(0,1 / 4)$, there is a constant $\Lambda$, independent of $t$, such that, if $\left|\lambda_{\omega}(t)\right| \geq \Lambda$, then

$$
\left|\lambda_{\omega}(t)-z_{n}(t)\right| \leq\left|\lambda_{\omega}(t)\right|^{(1 / 2)+\varepsilon,} \quad \text { for some } \quad n \in \mathbb{Z} .
$$

Using (40) and (41) the above estimate can be written as

$$
\left|\lambda_{\omega}(t)-z_{n}(0)\right| \leq K|n|^{2+4 \varepsilon}, \quad \text { for some } n \in \mathbb{Z} .
$$

Finally, since $\varepsilon \in(0,1 / 4)$, the estimates (42) and (44) make it impossible for $\lambda_{\omega}(t)$ to move continuously to infinity. Hence (32) is impossible and the theorem is proved for $a, \rho \in C^{\infty}(\mathbb{R})$. The general case follows by approximation by $C^{\infty}$ functions. We just have to observe that $D(\lambda)$ can be written in terms of the fundamental solutions $u_{j}(b ; \lambda)$ (and their derivatives) and apply the proposition of the appendix.

Remark 2. If $D\left(\lambda^{*}\right)=0$, then the theorem implies that $\lambda^{*}$ is in a gap or a $\psi$-gap. Thus, the corresponding Floquet multipliers $r_{j}\left(\lambda^{*}\right), j=1,2,3,4$, are all real (thus, $r_{j}\left(\lambda^{*}\right)=e^{i k_{j}}$ where $\Re\left\{k_{j}\right\}=0$ or $\left.\pi / b\right)$. As we have already seen (see also Lemma 4 below), if $\lambda^{*}$ is in a spectral gap, then we always have $r_{1}\left(\lambda^{*}\right), r_{4}\left(\lambda^{*}\right)>0$, while $r_{2}\left(\lambda^{*}\right), r_{3}\left(\lambda^{*}\right)$ may be positive or negative; however, if $\lambda^{*}$ is in a $\psi$-gap, then all $r_{j}\left(\lambda^{*}\right), j=1,2,3,4$, have the same sign. Furthermore, if $\lambda^{*}$ is in a gap or a $\psi$-gap, then (39) implies that $r_{j}^{\prime}\left(\lambda^{*}\right)=0$, for some $j=1,2,3,4$, and conversely.

The next theorem goes a little deeper. It completes Theorem 1.

Theorem 3. For a fixed $k \in \mathbb{C}$, the multiplicity $m$ of any zero $\lambda^{*}$ of $F(\lambda ; k)$ can be either one or two (of course, by Theorem $1, m=2$ if and only if $D\left(\lambda^{*}\right)=0$ ). 
Proof. Let $\lambda^{*}$ be a zero of $F(\lambda ; k)$ with multiplicity $m \geq 3$. Then (30) implies that $\lambda^{*}$ is a zero of $D(\lambda)$ of multiplicity at least $m-1$. But, by Theorem 2 , the multiplicity of any zeros of $D(\lambda)$ can be at most two. Thus, $m=3$ and $\lambda^{*}$ is a double zero of $D(\lambda)$. But then, again by Theorem 2 , we must have that $\lambda^{*}$ corresponds to a closed $\psi$-gap, i.e., it is a double zero of $E(\lambda)$; thus $E\left(\lambda^{*}\right)=$ $E^{\prime}\left(\lambda^{*}\right)=0$. Next we observe that, using (16), we can write (30) as

$$
A^{\prime}(\lambda)^{2} F(\lambda ; k)-\frac{E^{\prime}(\lambda)}{2} F_{\lambda}(\lambda ; k)-F_{\lambda}(\lambda ; k)^{2}=\frac{D(\lambda)}{16},
$$

which implies that $\lambda^{*}$ is a zero of $D(\lambda)$ of multiplicity 3 , a contradiction! Thus, $F(\lambda ; k)$ cannot have any zeros of multiplicity larger than two.

We continue with a lemma which is by itself interesting since it characterizes the zeros of the entire functions $A(\lambda)$ and $B(\lambda)$.

Lemma 2. (a) The set of zeros of $A(\lambda)$ of (4) is the $(\pi /(2 b))$-Floquet $\psi$-spectrum $\Psi_{\pi / 2 b}(a, \rho)$. Furthermore, all zeros of $A(\lambda)$ are simple.

(b) The set of zeros of $B(\lambda)$ of equality (9) is the $(\pi /(2 b))$-Floquet spectrum $\left\{\lambda_{n}(\pi /(2 b))\right\}_{n=1}^{\infty}$. Again, all zeros of $B(\lambda)$ are simple.

Proof. Part (a) follows immediately from (15) and Theorem C.

Part (b) also follows immediately from the fact (see (14)) that $B(\lambda)=$ $\left(r_{1}+r_{1}^{-1}\right)\left(r_{2}+r_{2}^{-1}\right)$.

Remark 3. The lemma implies that $B(\lambda)$ has one zero (counting multiplicities) in the interior of each band of the spectrum of (11) and no other zeros. In particular, all zeros of $B(\lambda)$ are (real and) strictly positive and simple.

Likewise $A(\lambda)$ has one zero (counting multiplicities) in the interior of each $\psi$ band of the $\psi$-spectrum of (1) and no other zeros. In particular, all zeros of $A(\lambda)$ are strictly negative and simple.

From Theorems 1, 2, and 3, it follows that, if $\lambda^{*}$ is a multiple zero of $F(\lambda ; k)$, then $\lambda^{*} \in \mathbb{R}$, its multiplicity is two, and we must have $k \in \mathbb{C}$ with $\Re\{k\}=0$ or $\pi / b$ (without loss of generality). Using Lemma 1 we can, in addition, prove the following:

Theorem 4. A given number $\lambda^{*}$ can be a double zero of $F(\lambda ; k)$ for at most one value of $k$ with $\Re\{k\} \in\{0, \pi / b\}$.

Proof. Assume

$$
F\left(\lambda^{*} ; k\right)=F_{\lambda}\left(\lambda^{*} ; k\right)=0 .
$$

Then (29) holds, namely

$$
B^{\prime}\left(\lambda^{*}\right)-2 A^{\prime}\left(\lambda^{*}\right) \cos (k b)=0 .
$$

If there are two distinct $k_{1} \neq k_{2}$ with $\Re\left\{k_{1}\right\}, \Re\left\{k_{2}\right\} \in\{0, \pi / b\}$, for which the above is true, we must have

$$
B^{\prime}\left(\lambda^{*}\right)=A^{\prime}\left(\lambda^{*}\right)=0 .
$$

Now the functions $A(\lambda)$ and $B(\lambda)$ are entire of order $1 / 4$. Since the zeros of $A(\lambda)$ are positive and the zeros of $B(\lambda)$ are negative (see Remark 3 above), it follows by a well-known theorem of complex analysis (see, e.g., 37]) that the zeros of $A^{\prime}(\lambda)$ are positive while the zeros of $B^{\prime}(\lambda)$ are negative. Thus, (45) is impossible. 
Remark 4 . Let $D\left(\lambda^{*}\right)=0$ where $\lambda^{*}$ is in an open gap or $\psi$-gap. Then by Theorems $1,2,3$, and 4 there is a unique $k \in \mathbb{C} \backslash \mathbb{R}$ with $\Re\{k\}=0$ or $\pi / b$ such that $\lambda^{*}$ is a double zero of $F(\lambda ; k)$. The four Floquet multipliers that correspond to $\lambda^{*}$ are distinct (since $\lambda^{*}$ lies in an open gap or $\psi$-gap) and one of them is $r=e^{i k b}$ (where $r \in \mathbb{R}, r \neq \pm 1$ ). Hence there is only one (up to linear independence, of course) Floquet solution $f(x)$ satisfying

$$
f(x+b)=r f(x) .
$$

We can thus say that, for this particular $k$, the algebraic multiplicity of $\lambda^{*}$ is two, but its geometric multiplicity is one!

If $\lambda^{*}$ corresponds to a closed gap, i.e., $\lambda^{*}$ is a double periodic or antiperiodic eigenvalue, then, by Theorem $2, D\left(\lambda^{*}\right)=0$ and hence $\lambda^{*}$ is a double zero of $F(\lambda ; k)$ for a unique (see Theorem 4) $k$ that equals 0 in the periodic case, or equals $\pi / b$ in the antiperiodic case. But now, by Theorem B, the geometric multiplicity of $\lambda^{*}$ is also two.

Finally, if $\lambda^{*}$ corresponds to a closed $\psi$-gap, then again, by Theorem $2, D\left(\lambda^{*}\right)=$ 0 and hence $\lambda^{*}$ is a double zero of $F(\lambda ; k)$ for a unique nonreal $k$ with real part in $\{0, \pi / b\}$. In this case though (a kind of exception of the exception), as we will see later (Theorem 8) there are two linearly independent Floquet solutions with multiplier $r=e^{i k b}$; thus, the geometric multiplicity of $\lambda^{*}$ is also two.

\section{A Closer LOOK at the Endpoints of the PSEUdogaps}

4.1. The operators $L_{k}$. Let $k \in \mathbb{C}$ be given. As we have already seen, the eigenvalues of the problem

$$
\left[a(x) u^{\prime \prime}(x)\right]^{\prime \prime}=\lambda \rho(x) u(x), \quad u^{(j)}(b)=r u^{(j)}(0), \quad j=0,1,2,3,
$$

where $r=e^{i k b}$, are the zeros of the function $F(\lambda ; k)$ defined in (8).

Problems such as (46) (almost always related to operators with periodic coefficients) have an equivalent formulation (see, e.g., 21]):

Let $L_{k}$ be the operator on $L_{\rho}^{2}(0, b)$ defined by

$$
L_{k} v=\rho(x)^{-1}(d / d x+i k)^{2}\left[a(x)(d / d x+i k)^{2} v\right],
$$

with periodic boundary conditions. Then $v(x)$ is an eigenfunction of $L_{k}$ with corresponding eigenvalue $\lambda$ if and only if $u(x)=e^{i k x} v(x)$ is an eigenfunction of (46) corresponding to the same eigenvalue $\lambda$ (in other words, $u(x)$ is a Floquet solution of (1) with a prescribed multiplier $\left.r=e^{i k b}\right)$.

The adjoint operator of $L_{k}$ is

$$
L_{k}^{*}=L_{\bar{k}} \text {. }
$$

In particular, $L_{k}$ is selfadjoint if and only if $k \in \mathbb{R}$. Let $G_{k}(x, y ; \lambda)$ be the Green function of $L_{k}$ and $\widetilde{G}_{k}(x, y ; \lambda)$ be the Green function of (46). We have

$$
\widetilde{G}_{k}(x, y ; \lambda)=e^{i k(x-y)} G_{k}(x, y ; \lambda) .
$$

It is well known (see, e.g., [9]) that $G_{k}(x, y ; \lambda)$ can be expressed as an expression which is entire in $\lambda$ divided by $F(\lambda ; k)$. In fact, $G_{k}(x, y ; \lambda)$ is meromorphic in $\lambda$ and its poles are the zeros of $F(\lambda ; k)$. If $\lambda^{*}$ is a double zero of $F(\lambda ; k)$, then $\lambda^{*}$ can be a simple or a double pole of $G_{k}(x, y ; \lambda)$. In the latter case (see [9]), $L_{k}$ and hence (46) may not possess a complete set of (proper) eigenfunctions. More precisely, 
apart from the eigenfunction, say $\phi^{*}(x)$, that corresponds to $\lambda^{*}$, there may be a generalized eigenfunction $\psi^{*}(x)$ :

$$
\left(L_{k}-\lambda^{*}\right) \psi^{*}(x)=\phi^{*}(x) .
$$

In fact, it is known that if $a(x) \rho(x) \equiv 1$ (thus the problem is essentially of second order), then the above situation can actually happen (see [20]). We expect this anomaly to arise in the general case too, as long as $\lambda^{*}$ is in an open gap or $\psi$-gap. These comments should be compared with Remark 4 above.

4.2. The endpoints of an open $\psi$-gap. The following theorem presents another case where an algebraic multiplicity is equal to the corresponding geometric. It can be viewed as a partial complement of Theorem B (in the sense that Theorem B is about endpoints of bands, while the theorem below is about endpoints of nontouching $\psi$-bands). The remaining case of touching $\psi$-bands (equivalently: closed $\psi$-gaps) is covered later by Theorem 8 .

Theorem 5. Let $(\alpha, \beta)$ be an open $\psi$-gap of (1) (i.e., $\alpha<\beta)$ and $\nu=\alpha$ or $\nu=\beta$. Then the Floquet matrix $T(\nu)$ is similar to the matrix

$$
\left(\begin{array}{cccc}
r_{1} & 1 & 0 & 0 \\
0 & r_{1} & 0 & 0 \\
0 & 0 & r_{1}^{-1} & 1 \\
0 & 0 & 0 & r_{1}^{-1}
\end{array}\right)
$$

In other words, the equation

$$
\left[a(x) u^{\prime \prime}(x)\right]^{\prime \prime}=\nu \rho(x) u(x)
$$

has exactly two linearly independent proper Floquet solutions, one with multiplier $r_{1}$ and one with $r_{1}^{-1}$.

Proof. Let $\nu=\alpha$ or $\beta$. Since $(\alpha, \beta)$ is a $\psi$-gap and $\alpha<\beta$, we have that $E(\nu)=0$, but $E^{\prime}(\nu) \neq 0$. Thus, by (28),

$$
D(\nu) \neq 0 \text {. }
$$

In the complex $\lambda$-plane consider the open disk $B_{\varepsilon}(\nu)$, i.e., with center $\nu$ and radius $\varepsilon$. We choose $\varepsilon>0$ small enough so that

$$
D(\lambda) \neq 0 \quad \text { if } \quad \lambda \in B_{\varepsilon}(\nu) .
$$

Let

$$
r_{1}(\nu)=e^{i k(\nu) b}=r_{2}(\nu),
$$

where $i k(\nu)$ or $i k(\nu)-(\pi / b)$ is real and, without loss of generality, strictly positive (if not, we consider $r_{j}^{-1}$ instead of $r_{j}$ ). From (4), (9), and (16),

$$
r_{j}(\lambda)+\frac{1}{r_{j}(\lambda)}=\frac{A(\lambda)}{2} \pm \frac{\sqrt{E(\lambda)}}{2}, \quad j=1 \text { or } 2,
$$

where $\sqrt{\cdot}$ denotes the principal branch of the square root function. If $\lambda \in B_{\varepsilon}(\nu)$, then $E(\lambda)=E_{1}(\lambda)(\lambda-\nu)$, where $E_{1}(\lambda) \neq 0$ in $B_{\varepsilon}(\nu)$. Hence the above formula can be written as

$$
r_{1}(\lambda)+\frac{1}{r_{1}(\lambda)}=\frac{A(\lambda)}{2}+\frac{\sqrt{E_{1}(\lambda)}}{2} \sqrt{\lambda-\nu}
$$


and

$$
r_{2}(\lambda)+\frac{1}{r_{2}(\lambda)}=\frac{A(\lambda)}{2}-\frac{\sqrt{E_{1}(\lambda)}}{2} \sqrt{\lambda-\nu} .
$$

Assuming $\left|r_{1}(\lambda)\right|,\left|r_{2}(\lambda)\right|>1$, the above equations give $r_{1}(\lambda)=e^{i k_{1}(\lambda) b}$ and $r_{2}(\lambda)=$ $e^{i k_{2}(\lambda) b}$ uniquely. Of course, $k_{1}(\lambda) \neq k_{2}(\lambda)$ if $\lambda \in B_{\varepsilon}(\nu), \lambda \neq \nu$.

If $\varepsilon$ is sufficiently small, Theorem 1 implies that $F(\lambda ; k(\nu))$ has exactly one zero in $B_{\varepsilon}(\nu)$, namely $\lambda=\nu$. In fact, there is a neighborhood $N$ of $k(\nu)$ such that, if $k \in N$, then $F(\lambda ; k)$ has exactly one zero (counting multiplicities) $\lambda=\lambda(k)$ in $B_{\varepsilon}(\nu)$. Furthermore, if $k \in N, k \neq k(\nu)$, then (1) has four proper Floquet solutions corresponding to this $\lambda=\lambda(k)$, with corresponding multipliers $r_{1}(\lambda)=$ $e^{i k b}, r_{1}(\lambda)^{-1}=e^{-i k b}, r_{2}(\lambda)$, and $r_{2}(\lambda)^{-1}$, where $r_{2}(\lambda)$ is given by (48). Therefore, for $k \in N, k \neq k(\nu)$, we must have (since one of these four Floquet solutions corresponds to an eigenfunction of $L_{k}$ )

$$
\frac{1}{2 \pi i} \int_{\partial B_{\varepsilon}(\nu)}\left[\int_{0}^{b} G_{k}(x, x ; \lambda) d x\right] d \lambda=1,
$$

where, as in the previous subsection, $G_{k}(x, y ; \lambda)$ is the Green function of $L_{k}$. Letting $k \rightarrow k(\nu)$ we obtain

$$
\frac{1}{2 \pi i} \int_{\partial B_{\varepsilon}(\nu)}\left[\int_{0}^{b} G_{k(\nu)}(x, x ; \lambda) d x\right] d \lambda=1,
$$

which says that there is only one (proper) Floquet solution corresponding to $r_{1}(\nu)=$ $r_{2}(\nu)=e^{i k(\nu) b}$ (if there were two Floquet solutions, the value of the integral in (48) would have been 2). Considering the adjoint case, which has an equivalent behavior (see 9, Ch. 12, Sec. 5), we can conclude that there is, also, only one Floquet solution corresponding to $r_{1}(\nu)^{-1}=r_{2}(\nu)^{-1}=e^{-i k(\nu) b}$.

Remark 5. If $E(\nu)=0, \nu \neq 0$ (remember that $\nu \in \mathbb{R}$ ), then, since $L_{k}$ and its adjoint $L_{k}^{*}$ have the same number of proper and generalized eigenfunctions corresponding to $\nu$ (see [9], Ch. 12, Sec. 5), it follows that $T(\nu)$ is similar to one of the following matrices:

$$
\left(\begin{array}{cccc}
r_{1} & 1 & 0 & 0 \\
0 & r_{1} & 0 & 0 \\
0 & 0 & r_{1}^{-1} & 1 \\
0 & 0 & 0 & r_{1}^{-1}
\end{array}\right), \quad\left(\begin{array}{cccc}
r_{1} & 0 & 0 & 0 \\
0 & r_{1} & 0 & 0 \\
0 & 0 & r_{1}^{-1} & 0 \\
0 & 0 & 0 & r_{1}^{-1}
\end{array}\right) .
$$

Theorem 5 covers the case where $\nu$ is a simple zero of $E(\lambda)$. The case where $\nu$ is a double zero of $E(\lambda)$, i.e., the corresponding $\psi$-gap is closed, is deeper, since in this case (49) becomes

$$
\frac{1}{2 \pi i} \int_{\partial B_{\varepsilon}(\nu)}\left[\int_{0}^{b} G_{k(\nu)}(x, x ; \lambda) d x\right] d \lambda=2 .
$$

Now, by Theorem $1, \nu$ is a double zero of $F(\lambda ; k(\nu))$. Consequently, the above equation says that either $L_{k(\nu)}$ has two proper linearly independent eigenfunctions and hence $T(\nu)$ is diagonalizable, or $L_{k(\nu)}$ has one proper and one generalized eigenfunction and hence $T(\nu)$ is not diagonalizable. Later, in Theorem 8, we will see that the latter can never happen, i.e., $T(\nu)$ is always diagonalizable. 


\section{A multipoint eigenvalue Problem}

In the Hill case, the Dirichlet spectrum $\left\{\mu_{n}\right\}_{n=1}^{\infty}$ (i.e., the eigenvalues corresponding to the boundary conditions $u(0)=u(b)=0$ ) plays an important role in the general spectral theory, especially in the formulation and solution of the inverse spectral problem. We propose the following multipoint problem as an analogue of Hill's Dirichlet problem for the Euler-Bernoulli case:

$$
\left[a(x) u^{\prime \prime}(x)\right]^{\prime \prime}=\lambda \rho(x) u(x), \quad u(0)=u(b)=u(2 b)=u(3 b)=0 .
$$

An eigenvalue of (50) is any value of $\lambda$ for which (50) has a nontrivial solution. We call such a solution an eigenfunction of (50).

Physically the problem (50) describes the vibration of a (periodic) beam fixed at four points.

Let $u_{j}(x ; \lambda), j=1,2,3,4$, be the fundamental solutions of (1). Since every solution of (1) is a linear combination of the fundamental solutions, it follows that $\lambda$ is an eigenvalue of (50) (that is, $\lambda$ is such that (50) has a nontrivial solution) if and only if $\lambda$ is a zero of the entire function

$$
H(\lambda) \stackrel{\text { def }}{=}\left|\begin{array}{cccc}
u_{1}(0 ; \lambda) & u_{2}(0 ; \lambda) & u_{3}(0 ; \lambda) & u_{4}(0 ; \lambda) \\
u_{1}(b ; \lambda) & u_{2}(b ; \lambda) & u_{3}(b ; \lambda) & u_{4}(b ; \lambda) \\
u_{1}(2 b ; \lambda) & u_{2}(2 b ; \lambda) & u_{3}(2 b ; \lambda) & u_{4}(2 b ; \lambda) \\
u_{1}(3 b ; \lambda) & u_{2}(3 b ; \lambda) & u_{3}(3 b ; \lambda) & u_{4}(3 b ; \lambda)
\end{array}\right| .
$$

But $u_{1}(0 ; \lambda)=1$ and $u_{2}(0 ; \lambda)=u_{3}(0 ; \lambda)=u_{4}(0 ; \lambda)=0$; therefore,

$$
H(\lambda)=\left|\begin{array}{ccc}
u_{2}(b ; \lambda) & u_{3}(b ; \lambda) & u_{4}(b ; \lambda) \\
u_{2}(2 b ; \lambda) & u_{3}(2 b ; \lambda) & u_{4}(2 b ; \lambda) \\
u_{2}(3 b ; \lambda) & u_{3}(3 b ; \lambda) & u_{4}(3 b ; \lambda)
\end{array}\right| .
$$

We can, thus, say that the spectrum of (50) is the set of zeros of $H(\lambda)$. In particular,

$$
H(0)=b^{4}\left(\int_{0}^{b} \frac{d x}{a(x)}\right)^{2} ;
$$

thus 0 is not an eigenvalue of (50). In the unperturbed case we have

$$
H_{0}(\lambda)=\frac{1}{\lambda^{3 / 2}} \sinh \left(\lambda^{1 / 4} b\right) \sin \left(\lambda^{1 / 4} b\right)\left[\cosh \left(\lambda^{1 / 4} b\right)-\cos \left(\lambda^{1 / 4} b\right)\right]^{2} .
$$

Notice that by (31),

$$
H_{0}(\lambda)=\frac{D_{0}(\lambda)}{16 b^{2}}
$$

We continue with some properties of (50) and its accompanying function $H(\lambda)$. But first we need some lemmas.

Lemma 3. (a) If there is a nontrivial function $u(x)$ such that

$$
\begin{gathered}
{\left[a(x) u^{\prime \prime}(x)\right]^{\prime \prime}=\lambda \rho(x) u(x), \quad b_{1}<x<b_{2},} \\
u\left(b_{1}\right)=u^{\prime}\left(b_{1}\right)=u\left(b_{2}\right)=u^{\prime}\left(b_{2}\right)=0,
\end{gathered}
$$

then $\lambda$ is real and strictly positive. 
(b) Likewise, if there is a nontrivial function $u(x)$ such that

$$
\begin{gathered}
{\left[a(x) u^{\prime \prime}(x)\right]^{\prime \prime}=\lambda \rho(x) u(x), \quad x \in(b, \infty) \quad(\text { or } \quad x \in(-\infty, b)),} \\
u(b)=u^{\prime}(b)=0, \quad u \in L^{2}(b, \infty) \quad\left(\text { resp. } \quad u \in L^{2}(-\infty, b)\right),
\end{gathered}
$$

then $\lambda$ is real and strictly positive.

Proof. (a) Multiplying the equation by $\overline{u(x)}$ and integrating yields

$$
\int_{b_{1}}^{b_{2}}\left[a(x) u^{\prime \prime}(x)\right]^{\prime \prime} \overline{u(x)} d x=\lambda \int_{b_{1}}^{b_{2}} \rho(x) u(x) \overline{u(x)} d x .
$$

Next, by applying integration by parts (twice) in the left-hand side and using the boundary conditions, we obtain

$$
\int_{b_{1}}^{b_{2}} a(x) u^{\prime \prime}(x) \overline{u^{\prime \prime}(x)} d x=\lambda \int_{b_{1}}^{b_{2}} \rho(x) u(x) \overline{u(x)} d x .
$$

The assumption that $u(x)$ is not trivial, together with the boundary conditions $u\left(b_{1}\right)=u^{\prime}\left(b_{1}\right)=0$, imply that $u(x)$ is not a linear function. Thus both integrals in the above formula are strictly positive (remember $a(x), \rho(x)>0$, for all $x$ ); hence $\lambda>0$.

(b) The proof of this part is very similar to the proof of part (a). Assume that $u \in$ $L^{2}(b, \infty)$. Since $u(x)$ is a linear combination of Floquet solutions (possibly including generalized ones), it follows that $u(x)$ and its derivatives decay exponentially, as $x \rightarrow \infty$; thus, we can apply again integration by parts and get

$$
\int_{b}^{\infty} a(x) u^{\prime \prime}(x) \overline{u^{\prime \prime}(x)} d x=\lambda \int_{b}^{\infty} \rho(x) u(x) \overline{u(x)} d x
$$

hence, again $\lambda>0$.

The following lemma is contained in 31. We include it here for the sake of completeness.

Lemma 4. Let $\lambda>0$. If the Floquet multipliers are indexed so that $\left|r_{1}\right| \geq\left|r_{2}\right| \geq$ $\left|r_{3}\right| \geq\left|r_{4}\right|$, then $\left(r_{3}=r_{2}^{-1}\right.$ and $)$

$$
r_{1}>\left|r_{2}\right| \geq\left|r_{3}\right|>r_{4}=r_{1}^{-1} \text {. }
$$

Furthermore, the Floquet solutions $f_{1}(x)$ and $f_{4}(x)$ corresponding to $r_{1}$ and $r_{4}$ never vanish.

Proof. If $\lambda>0, u_{1}(x ; \lambda)$, the first fundamental solution of (11), and $u_{1}(-x ; \lambda)$ are increasing when $x \geq 0$. They actually grow exponentially. If $\lambda$ is in the spectrum of (11), $\left|r_{2}\right|=\left|r_{3}\right|=1$ and thus (53) and the statement about $f_{1}(x)$ and $f_{4}(x)$ must be true (remember $f_{j}(x)=e^{w_{j} x} p_{j}(x)$, where $p_{j}(x)$ is $b$-periodic and $r_{j}=e^{w_{j} b}$ ); otherwise, there would not be any exponentially growing solutions. Similarly, if $\lambda$ is not in the spectrum, $r_{2}$ and $r_{3}=r_{2}^{-1}$ are real. If we take the period of (11) to be $2 b$, then the Floquet multipliers become

$$
r_{1}^{2} \geq r_{2}^{2}>r_{3}^{2} \geq r_{4}^{2}
$$

But the above inequalities become equalities only if $\lambda$ is a zero of $E(\lambda)$. Since $\lambda>0$ and the zeros of $E(\lambda)$ are nonpositive, we must have

$$
\left|r_{1}\right|>\left|r_{2}\right|>\left|r_{3}\right|>\left|r_{4}\right| \text {. }
$$


Now

$$
u_{1}(x)=c_{1} f_{1}(x)+c_{2} f_{2}(x)+c_{3} f_{3}(x)+c_{4} f_{4}(x) .
$$

Hence the exponential growth of $u_{1}(x)$ and $u_{1}(-x)$ implies that $r_{1}$ and $r_{4}$ are positive and $f_{1}(x)$ and $f_{4}(x)$ do not change sign.

The next theorem should be compared with the property of the Hill operator stating that the Dirichlet eigenvalues are simple and their corresponding eigenfunctions are Floquet solutions [39]. The case left open (namely when $\mu$ is also a simple periodic or antiperiodic eigenvalue) is covered later by Theorem 7 .

Theorem 6. Let $\mu$ be an eigenvalue of (50). If $V(\mu)$ denotes the corresponding eigenspace, namely the vector space of all eigenfunctions of (50) associated to $\mu$, then $\operatorname{dim} V(\mu)=1$ or 2 . Furthermore, $V(\mu)$ always contains a proper Floquet solution; if $\operatorname{dim} V(\mu)=2$, then $V(\mu)$ always contains two linearly independent proper Floquet solutions, except possibly in the case where $\mu$ is also a simple periodic or antiperiodic eigenvalue of (1) (in fact, we will see later, in Theorem 7, that this exception can never happen).

Proof. Let $H(\mu)=0$. Assume that $\operatorname{dim} V(\mu)=3$ (clearly $\operatorname{dim} V(\mu)<4)$. Then we have three linearly independent eigenfunctions $\phi_{1}(x), \phi_{2}(x)$, and $\phi_{3}(x)$ corresponding to $\mu$. Let

$$
\phi(x)=c_{1} \phi_{1}(x)+c_{2} \phi_{2}(x)+c_{3} \phi_{3}(x) .
$$

We have $\phi(0)=\phi(b)=0$. Also, we can choose $c_{1}, c_{2}$, and $c_{3}$ (not all zero) so that $\phi^{\prime}(0)=\phi^{\prime}(b)=0$. Hence, Lemma 3 implies that $\mu>0$. But then, Lemma 4 implies that the associated (to $\mu$ ) Floquet solutions $f_{1}(x)$ and $f_{4}(x)$ never vanish. Furthermore, the space spanned by $\phi_{1}(x), \phi_{2}(x)$, and $\phi_{3}(x)$ and the space spanned by $f_{1}(x)$ and $f_{4}(x)$ must have a nontrivial intersection. Thus there is an eigenfunction of (50) of the form

$$
\gamma_{1} f_{1}(x)+\gamma_{4} f_{4}(x) .
$$

But this implies easily that

$$
f_{1}(0 ; \mu) f_{4}(0 ; \mu)=0
$$

a contradiction. Thus $\operatorname{dim} V(\mu)<3$.

We now prove the rest of the theorem (see Theorem B and formula (16) for the definition of $F^{+}, F^{-}$, and $E$ that appear below).

Case $F^{+}(\mu) F^{-}(\mu) E(\mu) \neq 0$. Then, for $\lambda=\mu$, (1) possesses four distinct Floquet multipliers $r_{j}=r_{j}(\mu), j=1,2,3,4$; therefore, it has four linearly independent (proper) Floquet solutions $f_{j}(x)=f_{j}(x ; \mu), j=1,2,3,4$, with

$$
f_{j}(x+b)=r_{j} f_{j}(x) \text {. }
$$

If, in addition, $\mu$ is in the spectrum of (501), then

$$
\left|\begin{array}{cccc}
f_{1}(0) & f_{2}(0) & f_{3}(0) & f_{4}(0) \\
f_{1}(b) & f_{2}(b) & f_{3}(b) & f_{4}(b) \\
f_{1}(2 b) & f_{2}(2 b) & f_{3}(2 b) & f_{4}(2 b) \\
f_{1}(3 b) & f_{2}(3 b) & f_{3}(3 b) & f_{4}(3 b)
\end{array}\right|=0
$$


namely,

or

$$
f_{1}(0 ; \mu) f_{2}(0 ; \mu) f_{3}(0 ; \mu) f_{4}(0 ; \mu)\left|\begin{array}{cccc}
1 & 1 & 1 & 1 \\
r_{1} & r_{2} & r_{3} & r_{4} \\
r_{1}^{2} & r_{2}^{2} & r_{3}^{2} & r_{4}^{2} \\
r_{1}^{3} & r_{2}^{3} & r_{3}^{3} & r_{4}^{3}
\end{array}\right|=0
$$

$$
f_{1}(0 ; \mu) f_{2}(0 ; \mu) f_{3}(0 ; \mu) f_{4}(0 ; \mu) \prod_{1 \leq j<l \leq 4}\left(r_{l}-r_{j}\right)=0 .
$$

But the $r_{j}$ 's are distinct; thus,

$$
f_{1}(0 ; \mu) f_{2}(0 ; \mu) f_{3}(0 ; \mu) f_{4}(0 ; \mu)=0 .
$$

This means that some Floquet solution, say $f_{1}(x ; \mu)$, is an eigenfunction of (50).

Next, let $\phi(x)$ be another eigenfunction of (150) corresponding to $\mu$. That is, $\phi(x)$ and $f_{1}(x)$ are linearly independent. It follows that there is a constant $c_{1}$ such that $\widetilde{\phi}(x)=\phi(x)-c_{1} f_{1}(x)$ is an eigenfunction of (50) and

$$
\widetilde{\phi}(x)=c_{2} f_{2}(x)+c_{3} f_{3}(x)+c_{4} f_{4}(x) .
$$

But this implies easily that

$$
f_{2}(0 ; \mu) f_{3}(0 ; \mu) f_{4}(0 ; \mu)=0,
$$

which, again, means that some Floquet solution $f_{j}, j=2,3,4$, say $f_{2}(x ; \mu)$, is an eigenfunction of (50).

Case $E(\mu)=0$. That means that $\mu<0$ and $r_{1}=r_{2}=r_{3}^{-1}=r_{4}^{-1}$. If we have coexistence of two Floquet solutions $f_{1}(x)$ and $f_{2}(x)$ with multiplier $r_{1}$, then (see Remark 5) we also have coexistence of two Floquet solutions, $f_{3}(x)$ and $f_{4}(x)$, with multiplier $r_{1}^{-1}$. We can then find constants $c_{1}, c_{2}, c_{3}$, and $c_{4}$, such that $c_{1} f_{1}(x)+c_{2} f_{2}(x)$ and $c_{3} f_{3}(x)+c_{4} f_{4}(x)$ are in $V(\mu)$. If we do not have coexistence, then (see Remark 5) we have two proper Floquet solutions $f_{1}(x)$ and $f_{3}(x)$ with corresponding multipliers $r_{1}$ and $r_{1}^{-1}$, and two generalized Floquet solutions $g_{1}(x)$ and $g_{3}(x)$ satisfying

$$
g_{1}(x+b)=r_{1} g_{1}(x)+d_{1} f_{1}(x), \quad g_{3}(x+b)=r_{1}^{-1} g_{3}(x)+d_{3} f_{3}(x), \quad d_{1} d_{3} \neq 0 .
$$

Since $H(\mu)=0$, we must have

$$
\left|\begin{array}{cccc}
f_{1}(0) & g_{1}(0) & f_{3}(0) & g_{3}(0) \\
f_{1}(b) & g_{1}(b) & f_{3}(b) & g_{3}(b) \\
f_{1}(2 b) & g_{1}(2 b) & f_{3}(2 b) & g_{3}(2 b) \\
f_{1}(3 b) & g_{1}(3 b) & f_{3}(3 b) & g_{3}(3 b)
\end{array}\right|=0
$$

which implies

$$
f_{1}(0 ; \mu)^{2} f_{3}(0 ; \mu)^{2}=0 .
$$

The last equality says that one Floquet solution, say $f_{1}(x)$, is also an eigenfunction of (50). If $\phi(x)$ is another eigenfunction of (50) corresponding to $\mu$, then there is an eigenfunction of the form

$$
\widetilde{\phi}(x)=c_{1} g_{1}(x)+c_{3} f_{3}(x)+c_{4} g_{3}(x) .
$$

This implies that $g_{1}(x) \in V(\mu)$ or $f_{3}(x) \in V(\mu)$.

From (50) and the fact that $f_{1}(x)$ has the form

$$
f_{1}(x)=e^{w_{1} x} p_{1}(x) \quad \text { with } \quad r_{1}=e^{w_{1} b} \quad \text { and } \quad p_{1}(x+b)=p_{1}(x),
$$


it follows that $g_{1}(x)$ has the form

$$
g_{1}(x)=\left[p_{2}(x)+\beta p_{1}(x) x\right] e^{w_{1} x} \quad \text { with } \quad \beta=\frac{d_{1}}{r_{1} b} \quad \text { and } \quad p_{2}(x+b)=p_{2}(x) .
$$

Let us assume $g_{1}(x) \in V(\mu)$. Since we also have $g_{1}(x) \in V(\mu)$, it follows that $p_{1}(0)=p_{2}(0)=0$; therefore,

$$
f_{1}(n b)=g_{1}(n b)=0, \quad \text { for all } \quad n \in \mathbb{Z} .
$$

Next notice that

$$
f_{1}^{\prime}(n b)=r_{1}^{n} p_{1}^{\prime}(0) \neq 0 \quad \text { and } \quad g_{1}^{\prime}(n b)=r_{1}^{n}\left[n b p_{1}^{\prime}(0)+p_{2}^{\prime}(0)\right] \quad \text { for all } n \in \mathbb{Z},
$$

where $p_{1}^{\prime}(0) \neq 0$ follows from Lemma 3 . Introduce

$$
v(x)=p_{2}^{\prime}(0) f_{1}(x)-p_{1}^{\prime}(0) g_{1}(x),
$$

so that

$$
\left[a(x) v^{\prime \prime}(x)\right]^{\prime \prime}=\mu \rho(x) v(x)
$$

and (for all $n \in \mathbb{Z}$ )

$$
v(n b)=0, \quad v^{\prime}(0)=0 .
$$

By (59),

$$
\int_{0}^{n b}\left[a(x) v^{\prime \prime}(x)\right]^{\prime \prime} \overline{v(x)} d x=\mu \int_{0}^{n b} \rho(x) v(x) \overline{v(x)} d x .
$$

Then, integration by parts and (60) yield (recall that $a(n b)=a(0)$ )

$$
-a(0) v^{\prime \prime}(n b) \overline{v^{\prime}(n b)}+\int_{0}^{n b} a(x) v^{\prime \prime}(x) \overline{v^{\prime \prime}(x)} d x=\mu \int_{0}^{n b} \rho(x) v(x) \overline{v(x)} d x .
$$

If we set

$$
\varepsilon=\varepsilon\left(r_{1}\right)= \begin{cases}-1, & \text { if }\left|r_{1}\right|>1, \\ 1, & \text { if }\left|r_{1}\right|<1,\end{cases}
$$

then (56) and (57) imply that $f_{1}(x), g_{1}(x)$, and their derivatives decay exponentially, as $x \rightarrow \varepsilon \infty$, and by (58) the same is true for $v(x)$. Thus (61) implies that

$$
\int_{0}^{\varepsilon \infty} a(x) v^{\prime \prime}(x) \overline{v^{\prime \prime}(x)} d x=\mu \int_{0}^{\varepsilon \infty} \rho(x) v(x) \overline{v(x)} d x ;
$$

in particular, $\mu>0$, a contradiction. Therefore, $g_{1}(x) \notin V(\mu)$ and we are left with the only alternative, namely that $f_{3}(x) \in V(\mu)$.

Case $F^{+}(\mu) F^{-}(\mu)=0$. That means that $\mu>0$ is a periodic or antiperiodic eigenvalue. If we have coexistence of two periodic or antiperiodic Floquet solutions, then a linear combination of these can produce an eigenfunction of (50), and there is no other eigenfunction, i.e., $\operatorname{dim} V(\mu)=1$ (since, by Lemma 4 , the other two Floquet solutions never vanish). If there is only one periodic (or antiperiodic) Floquet solution, say $f_{2}(x)$, then there is a generalized Floquet solution $g_{2}(x)$, satisfying

$$
g_{2}(x+b)=\varepsilon g_{2}(x)+c_{2} f_{2}(x), \quad c_{2} \neq 0,
$$


where $\varepsilon=1$, if $F^{+}(\mu)=0$, and $\varepsilon=-1$, if $F^{-}(\mu)=0$. In this case, $H(\mu)=0$ implies that

$\left|\begin{array}{cccc}f_{1}(0) & f_{2}(0) & g_{2}(0) & f_{4}(0) \\ f_{1}(b) & f_{2}(b) & g_{2}(b) & f_{4}(b) \\ f_{1}(2 b) & f_{2}(2 b) & g_{2}(2 b) & f_{4}(2 b) \\ f_{1}(3 b) & f_{2}(3 b) & g_{2}(3 b) & f_{4}(3 b)\end{array}\right|=0$

which implies easily that

$$
f_{1}(0 ; \mu) f_{2}(0 ; \mu)^{2} f_{4}(0 ; \mu)=0
$$

hence there is a Floquet solution in $V(\mu)$. But, if $f_{2}(x) \in V(\mu)$ and $\operatorname{dim} V(\mu)=2$ (this is, however, impossible, as we will see later in Theorem 7 ), we cannot, for the moment, exclude the possibility that $g_{2}(x) \in V(\mu)$.

Remark 6 . One part of Theorem 6 states that the geometric multiplicity $m_{g}(\mu)$ of any eigenvalue $\mu$ of (50) cannot exceed two. We can define the algebraic multiplicity $m_{a}(\mu)$ of $\mu$ to be its multiplicity as a zero of $H(\lambda)$. From the above proof it follows easily that $m_{a}(\mu) \geq m_{g}(\mu)$. Theorems 7 and 8 below establish the equality of the two multiplicities.

The lemma that follows is needed for the proof of Theorem 7 below.

Lemma 5. There are no zeros of $H(\lambda)$ in the interior of the bands or in the interior of the $\psi$-bands.

Proof. Assume that $\mu$ is in the interior of a band. Then $F^{+}(\mu) F^{-}(\mu) E(\mu) \neq 0$; hence (54) must hold. By Lemma 4 we have that $f_{1}(x ; \mu)$ and $f_{4}(x ; \mu)$ never vanish,

$$
r_{1}(\mu)=r_{4}(\mu)^{-1}>1 \quad \text { and } \quad\left|r_{2}(\mu)\right|=\left|r_{3}(\mu)\right|=1,
$$

where $r_{3}(\mu)=r_{2}^{-1}(\mu)=\overline{r_{2}(\mu)} \notin \mathbb{R}$. Also, $f_{3}(x ; \mu)=\overline{f_{2}(x ; \mu)}$. Therefore,

$$
f_{2}(0 ; \mu)=f_{3}(0 ; \mu)=0 .
$$

We can, thus, write a linear combination

$$
f(x)=d_{2} f_{2}(x ; \mu)+d_{3} f_{3}(x ; \mu)
$$

such that $f(0)=f^{\prime}(0)=0$. This means that $f(x)$ can be written as a linear combination of the fundamental solutions $u_{3}(x ; \mu)$ and $u_{4}(x ; \mu)$, namely

$$
f(x)=\gamma_{3} u_{3}(x ; \mu)+\gamma_{4} u_{4}(x ; \mu) .
$$

But (see 31) $u_{3}(x ; \mu) \rightarrow \infty$, as $x \rightarrow \pm \infty$, while $u_{4}(x ; \mu) \rightarrow \pm \infty$, as $x \rightarrow \pm \infty$. Since $f(x)$ is bounded we can conclude that (63) is impossible. Thus (62) is impossible and $H(\mu) \neq 0$.

Assume now that $\mu$ is in the interior of a $\psi$-band (hence $\mu<0$ ). Then there is a $k \in(0, \pi / b)$ such that

$$
r_{1}(\mu)=\frac{1}{r_{4}(\mu)}=\overline{r_{2}(\mu)}=\frac{1}{\overline{r_{3}(\mu)}}=\left|r_{1}(\mu)\right| e^{i k b} .
$$

If $H(\mu)=0$, then (54) implies that $f_{j}(0 ; \mu)$, for some $j$. Let us assume that

$$
f_{1}(0 ; \mu)=0 \text {. }
$$

We have

$$
f_{1}(x ; \mu)=e^{\alpha x} e^{i k x} p_{1}(x) \quad \text { and } \quad f_{2}(x ; \mu)=\overline{f_{1}(x ; \mu)}=e^{\alpha x} e^{-i k x} \overline{p_{1}(x)},
$$


where $\alpha \in R \backslash\{0\}$ and $p_{1}(x+b)=p_{1}(x)$. Thus

$$
f_{2}(0 ; \mu)=0
$$

and $f_{1}(\cdot ; \mu), f_{2}(\cdot ; \mu) \in L^{2}(0, \infty)$ (if $\left.\alpha<0\right)$ or $f_{1}(\cdot ; \mu), f_{2}(\cdot ; \mu) \in L^{2}(-\infty, 0)$ (if $\alpha>0)$. We can, therefore, write a nontrivial linear combination

$$
u(x)=c_{1} f_{1}(x ; \mu)+c_{1} f_{1}(x ; \mu)
$$

that satisfies all assumptions of part (b) of Lemma 3 (with $b=0$ ). Hence $\mu>0$, a contradiction. Therefore, $H(\mu) \neq 0$.

We are now ready to prove our main theorem regarding the spectrum of (51), i.e., the zeros of $H(\lambda)$. The statement of the theorem resembles the one of Theorem 2 .

Theorem 7. All zeros of $H(\lambda)$, of (51), are real and they are located as follows: (a) $H(\lambda)$ has exactly one (simple) zero in the closure of each gap of the spectrum $S(a, \rho)$ (with the understanding that, if the gap is closed, i.e., collapses to a double periodic or antiperiodic eigenvalue, say $\lambda^{*}$, then the simple zero of $H(\lambda)$ is $\left.\lambda^{*}\right)$; (b) $H(\lambda)$ has exactly two zeros (counting multiplicities) in the closure of each $\psi$-gap of the pseudospectrum. In case (b), if the $\psi$-gap is closed, i.e., collapses to a point $\nu^{*}$, where $\nu^{*}=\nu_{2 n-1}=\nu_{2 n}$, or $\nu^{*}=\nu_{2 n-1}^{\prime}=\nu_{2 n}^{\prime}$, for some $n=1,2,3, \ldots$ (see the statement of Theorem D), then $\nu^{*}$ is a double zero of $H(\lambda)$. There are no other zeros of $H(\lambda)$.

Proof. As in the case of Theorem 2 (see also the appendix), we only need to prove the theorem for the case of smooth $a(x)$ and $\rho(x)$. Hence, from now on we assume $a, \rho \in C^{\infty}(\mathbb{R})$.

By (52) the theorem is valid in the unperturbed case $a(x) \equiv \rho(x) \equiv 1$. For general $a(x)$ and $\rho(x)$ let us consider the deformation (19) and the solutions $\phi_{j}(x ; \lambda ; t)$, $j=1,2,3,4$, satisfying (22). We set

$$
\widetilde{H}(\lambda ; t)=\left|\begin{array}{cccc}
\phi_{1}(0 ; t) & \phi_{2}(0 ; t) & \phi_{3}(0 ; t) & \phi_{4}(0 ; t) \\
\phi_{1}(b ; t) & \phi_{2}(b ; t) & \phi_{3}(b ; t) & \phi_{4}(b ; t) \\
\phi_{1}(2 b ; t) & \phi_{2}(2 b ; t) & \phi_{3}(2 b ; t) & \phi_{4}(2 b ; t) \\
\phi_{1}(3 b ; t) & \phi_{2}(3 b ; t) & \phi_{3}(3 b ; t) & \phi_{4}(3 b ; t)
\end{array}\right|,
$$

where the dependence of $\phi_{j}$ in $\lambda$ has been suppressed for typographical convenience. For small $|\lambda|$ 's, the $\phi_{j}$ 's can be chosen so that $\widetilde{H}(0 ; t)$ stays away from 0 , uniformly in $t$. Notice that $\widetilde{H}(\lambda ; t)$ and $H(\lambda ; t)$ have the same zeros. The multiplicities of their zeros also agree (this can be easily checked when $t=0$; then, since $t$ is moving continuously, the multiplicities of two corresponding (i.e., equal) zeros, one of $H$ and one of $\widetilde{H}$, cannot suddenly become different).

In order to use the technique described in Subsection 3.1, we first need to estimate the large-magnitude zeros of $\widetilde{H}(\lambda ; t)$. First, consider $\left\{\mu_{n}(0)\right\}_{n \in \mathbb{Z}}$, the set of zeros of $\widetilde{H}(\lambda ; 0)$, counting multiplicities. As we have seen, $\widetilde{H}(\lambda ; 0)$ and $D_{0}(\lambda)$ have the same zeros; thus, (35) gives

$$
\mu_{1-2 l}(0)=\mu_{-2 l}(0)=-4\left(\frac{l \pi}{b}\right)^{4}, \quad \mu_{2 l-1}(0)=\mu_{2 l}(0)=\left(\frac{l \pi}{b}\right)^{4}, \quad l=1,2,3, \ldots .
$$


If $\mu$ is a large-magnitude zero of $\widetilde{H}(\lambda ; t)$, then (22) and (64) imply that

$$
e^{\varepsilon_{j} \mu^{1 / 4} b}\left[1+O\left(\frac{1}{\mu^{1 / 4}}\right)\right]=e^{\varepsilon_{l} \mu^{1 / 4} b}\left[1+O\left(\frac{1}{\mu^{1 / 4}}\right)\right]
$$

(uniformly in $t$ ), where $\varepsilon_{j}, \varepsilon_{l} \in\{i,-1,-i, 1\}, \varepsilon_{j} \neq \varepsilon_{l}$. This, in turn, implies that there is a $K>0$ (independent of $t$ ) such that

$$
\left|\mu^{1 / 4}-\mu_{n}(0)^{1 / 4}\right| \leq \frac{K}{|\mu|^{1 / 4}}, \quad \text { for some } \quad n \in \mathbb{Z},
$$

from which it follows that

$$
\left|\mu-\mu_{n}(0)\right| \leq K n^{2}, \quad \text { for some } \quad n \in \mathbb{Z} .
$$

In other words, if $H(\mu ; t)=0$ and $|\mu|$ is sufficiently large, then there is an integer $n$ such that $\mu$ is within distance $K n^{2}$ from $\mu_{n}(0)$. On the other hand, as we have already seen in (42), there is a constant $C>0$ such that

$$
\mu_{n+2}(0)-\mu_{n}(0) \geq C|n|^{3}, \quad \text { for all } n \in \mathbb{Z} \text {. }
$$

Therefore, as in the proof of Theorem 2, no new zeros of $H(\lambda ; t)$ can come from infinity, as we move $t$.

Thus, by the above discussion and Lemma 5 , the only way in which the theorem can be violated is if some zeros of $H(\lambda ; t)$ become nonreal. As we start moving $t$, the zeros in the gaps (including closed gaps) are all simple. Thus, the zeros that can first leave the real axis (in pairs of complex conjugates, of course) are the zeros in the $\psi$-gaps.

Let $(\alpha, \beta)$ be a $\psi$-gap $(\alpha, \beta$ vary continuously with $t)$. For $\lambda$ in $[\alpha, \beta]$, let $r_{1}$, $r_{2}, r_{3}=r_{2}^{-1}$, and $r_{4}=r_{1}^{-1}$, with $\left|r_{1}\right| \geq\left|r_{2}\right|>1$, be the corresponding Floquet multipliers of

$$
\left[a(x ; t) u^{\prime \prime}(x)\right]^{\prime \prime}=\lambda \rho(x ; t) u(x) .
$$

Since $(\alpha, \beta)$ is a $\psi$-gap, there is a $\delta>0$, independent of $t$, such that

$$
\left|r_{1}\right| \geq\left|r_{2}\right| \geq 1+\delta
$$

Now let $D \subset \mathbb{C}$ be a domain (depending on $t$ ) such that $[\alpha, \beta] \subset D$. If $D$ is sufficiently small, then, as functions of $\lambda, f_{j}(x ; \lambda ; t)$ and, in particular, $f_{j}(0 ; \lambda ; t)$, $j=1,2,3,4$, are analytic in $D$, with the only singularities being the branch points $\alpha, \beta$ (if $\alpha \neq \beta$ ). As $\lambda$ moves around one of these branch points, $f_{1}(x ; \lambda)$ becomes $f_{2}(x ; \lambda)$ and $f_{4}(x ; \lambda)$ becomes $f_{3}(x ; \lambda)$.

Initially (i.e., when $t=0), \mu=\alpha=\beta$ is a double zero of $H(\lambda ; 0)$ and there are two (linearly independent) Floquet solutions $f_{1}(x ; \mu)$ and $f_{4}(x ; \mu)$, with multipliers $r_{1}$ and $r_{4}$ respectively, satisfying $f_{1}(0 ; \mu)=f_{4}(0 ; \mu)=0\left(f_{1}(x ; \mu)\right.$ and $f_{4}(x ; \mu)$ are eigenfunctions of (50) corresponding to the eigenvalue $\mu$ ). As we move $t, f_{1}(0 ; \lambda)$ (or $f_{2}(0 ; \lambda)$, if we encounter a branch point) will continue to have a zero in $[\alpha, \beta]$, and so will $f_{4}(0 ; \lambda)$ (or $f_{3}(0 ; \lambda)$, if we encounter a branch point). In order for a zero of $H(\lambda ; t)$ to escape from the real axis, it first has to become double. So let us assume that, for $t=t_{0}, \mu=\mu\left(t_{0}\right) \in \mathbb{R}$ satisfies $f_{j}\left(0 ; \mu\left(t_{0}\right)\right)=f_{l}\left(0 ; \mu\left(t_{0}\right)\right)=0, j \neq l$, i.e., $\mu\left(t_{0}\right)$ is a double zero of $H\left(\lambda ; t_{0}\right)$. Notice that $f_{j}(x)$ and $f_{l}(x)$ must belong, one to $L^{2}(-\infty, 0)$ and one to $L^{2}(0, \infty)$. This follows by continuity, but, also, by Lemma 3 (if both $f_{j}(x)$ and $f_{l}(x)$ are, say, in $L^{2}(0, \infty)$, then we can construct a nontrivial solution $v(x)=c_{j} f_{j}(x)+c_{l} f_{l}(x)$, such that $v(0)=v^{\prime}(0)=0$ and $v \in L^{2}(0, \infty)$, which implies that $\mu\left(t_{0}\right)>0$, a contradiction). Thus, without loss 
of generality, we can take $j=1$ and $l=4$. Assume that, as $t$ gets larger than $t_{0}$, then immediately $\mu\left(t_{0}\right)$ splits into two nonreal zeros $\mu(t)$ and $\overline{\mu(t)}$ of $H(\lambda ; t)$. The corresponding Floquet solutions are $f_{1}(x ; \mu(t))$ (or $f_{2}(x ; \mu(t))$, if $\mu\left(t_{0}\right)$ is a branch point) and $f_{4}(x ; \overline{\mu(t)})$ (or $f_{3}(x ; \overline{\mu(t)})$, if $\mu\left(t_{0}\right)$ is a branch point). They also have to be complex conjugates. But this is a contradiction, since, on the one hand, $f_{1}(x)$, $f_{2}(x)$ are in $L^{2}(-\infty, 0)$ and $f_{3}(x), f_{4}(x)$ are in $L^{2}(0, \infty$,$) ; but, on the other hand, a$ function and its complex conjugate are in the same $L^{2}$-space. Therefore, the zeros of $H(\lambda ; t)$ can never leave the real axis and the theorem is proved.

Remark 7. Since, by the above theorem, all the positive zeros of $H(\lambda)$ are simple, the case in Theorem 6 that was left unanswered, namely when $\mu$ is also a simple periodic or antiperiodic eigenvalue, is now decided: We always have $\operatorname{dim} V(\mu)=1$.

Remark 8. If the coefficients of (1) are even functions, namely if

$$
a(-x)=a(x) \quad \text { and } \quad \rho(-x)=\rho(x),
$$

then for every solution $u(x)$ of (1) we have that $v(x)=u(-x)$ is also a solution. In particular, if $f(x)=e^{w x} p(x)$ is a Floquet solution, so is $f(-x)=e^{-w x} p(-x)$. It follows that, in this case, the positive zeros of $H(\lambda)$, i.e., the positive eigenvalues of (50), must also be periodic or antiperiodic eigenvalues, while the negative zeros of $H(\lambda)$ are all double, since, if $\mu<0$ is such that $H(\mu)=0$, then there are two eigenfunctions of (50), $f(x)=e^{w x} p(x)$ and $f(-x)=e^{-w x} p(-x)$ (one in $L^{2}(-\infty, 0)$ and the other in $\left.L^{2}(0, \infty)\right)$.

Remark 9. For $\xi \in \mathbb{R}$, consider the one-parameter family of shifted or translated functions $a_{\xi}(x)$ and $\rho_{\xi}(x)$ of $a(x)$ and $\rho(x)$, namely

$$
a_{\xi}(x)=a(x+\xi) \quad \text { and } \quad \rho_{\xi}(x)=\rho(x+\xi),
$$

and let $T(\lambda ; \xi)$ be the corresponding Floquet matrices. Then $T(\lambda ; \xi)$ is similar to $T(\lambda)$, for all $\xi$. In particular, this one-parameter family is isospectral and "isopseudospectral". On the other hand, the spectrum $\left\{\mu_{n}(\xi)\right\}_{n \in \mathbb{Z}}$ of (50) evolves with $\xi$ (thus we have an isospectral and iso-pseudospectral flow). It will be interesting to understand the evolution of $\mu_{n}(\xi)$ 's with $\xi$, since this might provide the solution to the inverse spectral problem. More generally, we would like to do the analysis of (11) from the point of view of 12. One relevant observation here is that, if $(\alpha, \beta)$ is a $\psi$-gap and $\mu$ is in $[\alpha, \beta]$, then there is a $\xi$ such that $\mu$ is in the spectrum of the multipoint problem for $a_{\xi}(x)$ and $\rho_{\xi}(x)$. This is because, if $\lambda \in[\alpha, \beta]$, then (1) always has a Floquet solution, say $f_{j}(x ; \lambda)$, that vanishes for some $x=x_{0}$. Taking $\xi=x_{0}$ does the job.

We believe that the same is true for the gaps of the spectrum; namely, that for any $\lambda$ in a gap, there is a $\xi$ so that $\lambda$ is in the spectrum of the multipoint problem for some $a_{\xi}(x)$ and $\rho_{\xi}(x)$.

The last theorem of this article is an application of Theorems 6 and 7. It completes Theorem 5 , so that the two theorems together form the analogue of Theorem B for the pseudospectrum.

Theorem 8. Let $\nu^{*}=\nu_{2 n-1}^{\prime}=\nu_{2 n}^{\prime}$ or $\nu^{*}=\nu_{2 n-1}=\nu_{2 n}$; namely, $\nu^{*}$ is a collapsed (i.e., closed) $\psi$-gap of (11) (equivalently, $\nu^{*}$ is a double zero of $E(\lambda)$-see Theorem 
$D)$. Then the Floquet matrix $T\left(\nu^{*}\right)$ is similar to the diagonal matrix

$$
\left(\begin{array}{cccc}
r_{1} & 0 & 0 & 0 \\
0 & r_{1} & 0 & 0 \\
0 & 0 & r_{1}^{-1} & 0 \\
0 & 0 & 0 & r_{1}^{-1}
\end{array}\right) .
$$

In other words, for the equation

$$
\left[a(x) u^{\prime \prime}(x)\right]^{\prime \prime}=\nu^{*} \rho(x) u(x),
$$

we have coexistence of four Floquet solutions, two with multiplier $r_{1}$ and two with multiplier $r_{1}^{-1}$.

Proof. Consider the shifts $a_{\xi}(x)$ and $\rho_{\xi}(x)$ of $a(x)$ and $\rho(x)$ of ([65). As we observed in Remark 9, the equation

$$
\left[a_{\xi}(x) u^{\prime \prime}(x)\right]^{\prime \prime}=\lambda \rho_{\xi}(x) u(x)
$$

has the same spectrum and pseudospectrum as (11), for all $\xi \in \mathbb{R}$. In particular, $\nu^{*}$ is a closed $\psi$-gap of (66), for all $\xi \in \mathbb{R}$. The Floquet multipliers $r_{j}=r_{j}\left(\nu^{*}\right)$, $j=1,2,3,4$, are the same for all $\xi \in \mathbb{R}$ and we have

$$
r_{1}=r_{2}=r_{3}^{-1}=r_{4}^{-1} \text {. }
$$

Next we consider the multipoint eigenvalue problem

$$
\left[a_{\xi}(x) u^{\prime \prime}(x)\right]^{\prime \prime}=\lambda \rho_{\xi}(x) u(x), \quad u(0)=u(b)=u(2 b)=u(3 b)=0 .
$$

From the above discussion and Theorem 7 it follows that $\nu^{*}$ is a double eigenvalue of (67), for all $\xi \in \mathbb{R}$. Thus, by Theorem 6 , for any $\xi \in \mathbb{R}$, there are two Floquet solutions, $f_{1, \xi}\left(x ; \nu^{*}\right)$ and $f_{4, \xi}\left(x ; \nu^{*}\right)$ of ([66), with corresponding multipliers $r_{1}$ and $r_{4}=r_{1}^{-1}$ such that

$$
f_{1, \xi}\left(0 ; \nu^{*}\right)=f_{4, \xi}\left(0 ; \nu^{*}\right)=0 .
$$

Each Floquet solution of (66) is a $\xi$-shift of a Floquet solution $f_{j}(x ; \lambda)$ of (1):

$$
f_{1, \xi}\left(x ; \nu^{*}\right)=f_{1}\left(x+\xi ; \nu^{*}\right) \quad \text { and } \quad f_{4, \xi}\left(x ; \nu^{*}\right)=f_{1}\left(x+\xi ; \nu^{*}\right) .
$$

Hence, if we did not have coexistence, then (68) would imply

$$
f_{1}\left(\xi ; \nu^{*}\right)=f_{4}\left(\xi ; \nu^{*}\right)=0, \quad \text { for all } \xi \in \mathbb{R},
$$

which is impossible since the Floquet solutions considered are nontrivial. Thus we have coexistence of two (linearly independent) Floquet solutions with multiplier $r_{1}$, and two Floquet solutions with multiplier $r_{1}^{-1}$.

Remark 10. The above theorem, together with Theorems 6 and 7, imply, in particular, that $m_{g}(\mu)=m_{a}(\mu)$, for any eigenvalue $\mu$ of (50) (see Remark 6).

In relation to Theorem 8 we notice that, if for some $\nu^{*}$ the Floquet matrix $T\left(\nu^{*}\right)$ is similar to the diagonal matrix $\operatorname{diag}\left(r_{1}, r_{1}, r_{1}^{-1}, r_{1}^{-1}\right)$, then $T\left(\nu^{*}\right)$ has a very special structure. Its sixteen entries must satisfy various relations.

Finally, we want to mention a conjecture and three open questions.

Conjecture. If all nonzero zeros of $E(\lambda)$ are double (see Theorem D), then $\rho(x) a(x) \equiv 1$. Equivalently: if all the $\psi$-gaps are closed, then the beam operator is a perfect square of a Hill-type operator. 
Open Question 1. An interesting question is: What can be said about $a(x)$ and $\rho(x)$ if we know that the spectrum $S(a, \rho)$ of (1) has no gaps; namely, if $S(a, \rho)=[0, \infty)$ ?

Open Question 2. In the Hill case there is a simple correspondence between periodic inverse spectral data and inverse spectral data of the separated boundary value problem on the interval $(0, b)$, where $b$ is the period of the potential (see [14]). Find the analogous correspondence for the beam operator. This will be a major step in the solution of the inverse periodic spectral problem for the beam.

Open Question 3. Extend the results presented in this paper to $n$-th order operators.

\section{APPENDIX}

We present here a proposition which was used in the proofs of some of our theorems.

Proposition. For $a_{n}, \rho_{n} \in C^{\infty}[0, b], a_{n}(x), \rho_{n}(x) \geq m>0, n=1,2,3, \ldots$, consider the initial value problems

$$
\begin{gathered}
{\left[a_{n}(x) u_{n}^{\prime \prime}(x)\right]^{\prime \prime}=\lambda \rho_{n}(x) u_{n}(x), \quad 0<x<b,} \\
u_{n}(0 ; \lambda)=\alpha, \quad u_{n}^{\prime}(0 ; \lambda)=\beta, \quad u_{n}^{\prime \prime}(0 ; \lambda)=\gamma, \quad u_{n}^{\prime \prime \prime}(0 ; \lambda)=\delta,
\end{gathered}
$$

where primes denote derivatives with respect to $x$ and $\lambda \in \mathbb{C}$ is a parameter. If

$$
a_{n}(x) \stackrel{C^{2}}{\rightarrow} a(x) \quad \text { and } \quad \rho_{n}(x) \stackrel{C}{\rightarrow} \rho(x), \quad x \in[0, b],
$$

then

$$
u_{n}(b ; \lambda) \rightarrow u(b ; \lambda)
$$

uniformly on compact subsets of $\mathbb{C}$, where

$$
\begin{gathered}
{\left[a(x) u^{\prime \prime}(x)\right]^{\prime \prime}=\lambda \rho(x) u(x),} \\
u(0 ; \lambda)=\alpha, \quad u^{\prime}(0 ; \lambda)=\beta, \quad u^{\prime \prime}(0 ; \lambda)=\gamma, \quad u^{\prime \prime \prime}(0 ; \lambda)=\delta .
\end{gathered}
$$

Proof. We write (69) as a first-order system

$$
\frac{d y_{n}}{d x}=A_{n}(x ; \lambda) y_{n}, \quad 0<x<b,
$$

where

$$
y_{n}(x ; \lambda)=\left(\begin{array}{c}
u_{n}(x ; \lambda) \\
u_{n}^{\prime}(x ; \lambda) \\
a_{n}(x) u_{n}^{\prime \prime}(x ; \lambda) \\
{\left[a_{n}(x) u_{n}^{\prime \prime}(x ; \lambda)\right]^{\prime}}
\end{array}\right), \quad A_{n}(x ; \lambda)=\left(\begin{array}{cccc}
0 & 1 & 0 & 0 \\
0 & 0 & a_{n}(x)^{-1} & 0 \\
0 & 0 & 0 & 1 \\
\lambda \rho_{n}(x) & 0 & 0 & 0
\end{array}\right) .
$$

The initial conditions (70) become

$$
y_{n}(0 ; \lambda)=\left(\begin{array}{c}
\alpha \\
\beta \\
a_{n}(0) \gamma \\
a_{n}^{\prime}(0) \gamma+a_{n}(0) \delta
\end{array}\right) .
$$

Consider also the problem

$$
\begin{gathered}
\frac{d y}{d x}=A(x ; \lambda) y, \quad 0<x<b, \\
y(0 ; \lambda)=y_{n}(0 ; \lambda),
\end{gathered}
$$


where

$$
A(x ; \lambda)=\lim _{n} A_{n}(x ; \lambda)=\left(\begin{array}{cccc}
0 & 1 & 0 & 0 \\
0 & 0 & a(x)^{-1} & 0 \\
0 & 0 & 0 & 1 \\
\lambda \rho(x) & 0 & 0 & 0
\end{array}\right) .
$$

Let us assume that $|\lambda| \leq M$, where $M$ is a fixed positive number. If we set

$$
w_{n}(x ; \lambda)=y_{n}(x ; \lambda)-y(x ; \lambda),
$$

then by (71), (72), and (73),

$$
\begin{aligned}
\frac{d w_{n}}{d x}=A_{n} y_{n}-A y= & A_{n} w_{n}+\left(A_{n}-A\right) y, \quad 0<x<b, \\
& w_{n}(0 ; \lambda)=0 .
\end{aligned}
$$

It follows that

$$
w_{n}(x ; \lambda)=\int_{0}^{x} A_{n}(\xi ; \lambda) w_{n}(\xi ; \lambda) d \xi+\int_{0}^{x}\left[A_{n}(\xi ; \lambda)-A(\xi ; \lambda)\right] y(\xi ; \lambda) d \xi .
$$

Thus

$$
\left|w_{n}(x ; \lambda)\right| \leq \varepsilon_{n}+\int_{0}^{x}\left\|A_{n}(\xi ; \lambda)\right\|\left|w_{n}(\xi ; \lambda)\right| d \xi
$$

where

$$
\varepsilon_{n}=\int_{0}^{b}\left\|A_{n}(\xi ; \lambda)-A(\xi ; \lambda)\right\||y(\xi ; \lambda)| d \xi
$$

Notice that

$$
\lim _{n} \varepsilon_{n}=0 .
$$

Applying the Gronwall inequality (see [9, Ch. 1, Prob. 1) to (74) we get

$$
\left|w_{n}(b ; \lambda)\right| \leq \varepsilon_{n}+\varepsilon_{n} \int_{0}^{b}\left\|A_{n}(\xi ; \lambda)\right\| e^{\int_{\xi}^{b}\left\|A_{n}(x ; \lambda)\right\| d x} d \xi
$$

and $\left\|A_{n}(x ; \lambda)\right\|$ is bounded for $x \in[0, b]$ and $|\lambda| \leq M$ (uniformly in $n$ ). Therefore, by (75) we have that

$$
w_{n}(b ; \lambda)=y_{n}(b ; \lambda)-y(b ; \lambda) \rightarrow 0
$$

uniformly in $\lambda$, as long as $|\lambda| \leq M$. Since $M$ is arbitrary, the proof is complete.

\section{ACKNowledGments}

The author wishes to express his gratitude to Professors Peter Kuchment and Sergey Novikov for helpful discussions and suggested references. We also want to thank Professor George Papanicolaou and the (NSF-funded) Mathematical Geophysics Summer School, held at Stanford University, for the great hospitality and the partial support of this work. Finally, the author thanks the anonymous referee for numerous constructive suggestions and comments. 


\section{REFERENCES}

[1] J. E. Avron and B. Simon, Analytic Properties of Band Functions, Annals of Physics, 110 (1978), 85-101. MR 57:14992

[2] A. Badanin and E. Korotyaev, Quasimomentum of Fourth Order Periodic Operator, preprint, 2001.

[3] V. Barcilon, Inverse Problem for a Vibrating Beam in the Free-Clamped Configuration, Philosophical Transactions of the Royal Society of London, Series A, 304 (1982), 211-251.

[4] R. Beals and R. R. Coifman, Scattering and Inverse Scattering for First Order Systems, Comm. Pure Appl. Math. 37, no. 1 (1984), 39-90. MR 85f:34020

[5] R. Beals, P. Deift, and C. Tomei, Direct and Inverse Scattering on the Line, Mathematical Surveys and Monographs, 28. American Mathematical Society, Providence, RI, 1988, xiv + 209 pp. MR 90a:58064

[6] R. CARlson, Compactness of Floquet Isospectral Sets for the Matrix Hill's Equation, Proceedings of the American Mathematical Society 128, no. 10 (2000), 2933-2941. MR 2000m:34027

[7] R. CARlson, Eigenvalue Estimates and Trace Formulas for the Matrix Hill's Equation, Journal of Differential Equations 167, no. 1 (2000), 211-244. MR 2001e:34157

[8] L. F. Caudill, P. A. Perry, And A. W. Schueller, Isospectral Sets for Fourth-Order Ordinary Differential Operators, SIAM J. Math. Anal. 29 (1998), 935-966. MR 99c:34022

[9] E. A. Coddington And N. Levinson, "Theory of Ordinary Differential Equations", Robert E. Krieger Publishing Company, Malabar, Florida, 1987. MR 16:1022b

[10] W. CRaig, The Trace Formula for Schrödinger Operators on the Line, Commun. Math. Phys. 126 (1989), 379-407. MR 90m:47063

[11] B. A. Dubrovin, I. M. Krichever, And S. P. Novikov, Integrable Systems. I, Dynamical Systems, IV, 177-332, Encyclopaedia of Mathematical Sciences, 4, Springer-Verlag, Berlin, 2001. MR 87k:58112

[12] B. A. Dubrovin, V. Matveev, And S. P. Novikov, Nonlinear Equations of KortewegdeVries Type, Finite Zone Linear Operators, and Abelian Varieties, Uspekhi. Mat. Nauk, 31 (1976), 55-136; Russian Math. Surveys, 31 (1976), 59-146. MR 55:899

[13] N. Dunford and J. T. Schwartz, "Linear Operators. Part II: Spectral Theory; Self Adjoint Operators in Hilbert Space", Wiley Classics Library Edition, New York, 1988. MR 90g:47001b

[14] A Finkel, E. Isaacson, and E. Trubowitz, An Explicit Solution of the Inverse Periodic Problem Hill's Equation, SIAM J. Math. Anal. 18, No. 1 (Jan. 1987), 46-53. MR 88d:34037

[15] F. Gesztesy, H. Holden, B. Simon, and Z. Zhao, Trace Formulae and Inverse Spectral Theory for Schrödinger Operators, Bull. Amer. Math. Soc. (New Series) 29 (1993), 250-255. MR 94c:34127

[16] F. Gesztesy and R. Weikard, Floquet Theory Revisited, in "Differential Equations and Mathematical Physics", Proceedings of the International Conference, Univ. of Alabama at Birmingham, March 13-17, 1994, International Press, 1995. MR 2000i:34163

[17] G. M. L. Gladwell, "Inverse Problems in Vibration," Martinus Nijhoff Publishers, Boston, 1986. MR 88b:73002

[18] R. Johnson, $m$-Functions and Floquet Exponents for Linear Differential Systems, Annali di Mathematica pura ed applicata (IV), CXLVII (1987), 211-248. MR 88m:34021

[19] R. Johnson and J. Moser, The Rotation Number for Almost Periodic Potentials, Comm. Math. Phys. 84 (1982), 403-438; erratum: Comm. Math. Phys. 90 (1983), 317-318. MR 83h:34018

[20] W. Konn, Analytic Properties of Bloch Waves and Wannier Functions, Annals of Physical Review, 115, no. 4 (August 1959), 809-821. MR 21:7000

[21] P. Kuchment, "Floquet Theory for Partial Differential Equations", Birkhäuser-Verlag, Basel, 1993. MR 94h:35002

[22] W. Magnus and S. Winkler, "Hill's Equation", Dover Publications, Inc., New York, 1979. MR 80k:34001

[23] M. M. Malamud, Necessary Conditions for the Existence of a Transformation Operator for Higher Order Equations, Funksional. Anal. i Ego Philozhen. 16 (1982), 74-75. Functional Anal. Appl. 16 (1982), 219-221. MR 84i:34011 
[24] H. P. McKean and E. Trubowitz, Hill's Operator and Hyperelliptic Function Theory in the Presence of Infinitely Many Branch Points, Comm. Pure Appl. Math. 29, no. 2 (1976), 143-226. MR 55:761

[25] H. P. McKean and P. van Moerbeke, The Spectrum of Hill's Equation, Invent. Math. 30, no. 3 (1975), 217-274. MR 53:936

[26] J. R. McLaughlin, On Constructing Solutions to an Inverse Euler-Bernoulli Problem, in "Inverse Problems of Acoustic and Elastic Waves", pp. 341-347, F. Santosa, et al. (editors), Philadelphia: SIAM, 1984. MR 86e:00016

[27] J. R. McLaughlin, Analytical Methods for Recovering Coefficients in Differential Equations from Spectral Data, SIAM Review 28 (1986), 53-72. MR 87d:34034

[28] R. E. Miller, The Eigenvalue Problem for a Class of Long, Thin Elastic Structures with Periodic Geometry, Quarterly of Applied Mathematics, LII, No. 2 (June 1994), 261-282. MR 95c:73008

[29] M. A. Naimark, "Linear Differential Operators", Parts I \& II, Frederick Ungar Publishing Co., New York, 1967 \& 1968. MR 35:6885, MR 41:7485

[30] S. P. Novikov, private communication (April 2001).

[31] V. G. Papanicolaou, The Spectral Theory of the Vibrating Periodic Beam, Comm. Math. Phys. 170 (1995), 359-373. MR 96d:34108

[32] V. G. Papanicolaou and D. Kravvaritis, An Inverse Spectral Problem for the EulerBernoulli Equation for the Vibrating Beam, Inverse Problems 13 (1997), 1083-1092. MR 98f:34016

[33] V. G. Papanicolaou and D. Kravvaritis, The Floquet Theory of the Periodic EulerBernoulli Equation, Journal of Differential Equations 150 (1998), 24-41. MR 2000a:34167

[34] M. Reed And B. Simon, "Methods of Modern Mathematical Physics, VI: Analysis of Operators", Academic Press, New York, 1978. MR 58:12429c

[35] L. A. Sachnovich, Inverse Problems for Differential Equations of Order $n>2$ with Analytic Coefficients, Matematicheskii Sbornik 46 (1958), 61-76. MR 20:5912

[36] S. Timoshenko and D. H. Young, "Elements of Strength of Materials", 5th Edition, D. Van Nostrand Company, Inc., Princeton, NJ, 1968.

[37] E. C. Titchmarsh, "The Theory of Functions", Second Edition, Oxford University Press, 1939.

[38] E. Trubowitz, The Inverse Problem for Periodic Potentials, Comm. Pure Appl. Math. 30, no. 3 (1977), 321-337. MR 55:3408

[39] S. VenaKides, private communication.

Department of Mathematics and Statistics, Wichita State University, Wichita, KANSAS 67260-0033

Current address: Department of Mathematics, National Technical University of Athens, Zografou Campus, 157 80, Athens, Greece

E-mail address: papanico@math.ntua.gr 